\title{
Reinterpreting aircraft measurements in anisotropic scaling turbulence
}

\author{
S. Lovejoy ${ }^{1}$, A. F. Tuck ${ }^{2}$, D. Schertzer ${ }^{3,4}$, and S. J. Hovde ${ }^{2}$ \\ ${ }^{1}$ Physics, McGill University, 3600 University st., Montreal, Que. H3A 2T8, Canada \\ ${ }^{2}$ NOAA Earth System Research Laboratory, Chemical Sciences Division, 325 Broadway, Boulder CO 80305-3337, USA \\ ${ }^{3}$ CEREVE, Université Paris Est, France \\ ${ }^{4}$ Météo France, 1 Quai Branly, Paris 75005, France
}

Received: 5 November 2008 - Published in Atmos. Chem. Phys. Discuss.: 4 February 2009

Revised: 14 May 2009 - Accepted: 30 June 2009 - Published: 27 July 2009

\begin{abstract}
Due to both systematic and turbulent induced vertical fluctuations, the interpretation of atmospheric aircraft measurements requires a theory of turbulence. Until now virtually all the relevant theories have been isotropic or "quasi isotropic" in the sense that their exponents are the same in all directions. However almost all the available data on the vertical structure shows that it is scaling but with exponents different from the horizontal: the turbulence is scaling but anisotropic. In this paper, we show how such turbulence can lead to spurious breaks in the scaling and to the spurious appearance of the vertical scaling exponent at large horizontal lags.

We demonstrate this using 16 legs of Gulfstream 4 aircraft near the top of the troposphere following isobars each between 500 and $3200 \mathrm{~km}$ in length. First we show that over wide ranges of scale, the horizontal spectra of the aircraft altitude are nearly $k^{-5 / 3}$. In addition, we show that the altitude and pressure fluctuations along these fractal trajectories have a high degree of coherence with the measured wind (especially with its longitudinal component). There is also a strong phase relation between the altitude, pressure and wind fluctuations; for scales less than $\approx 40 \mathrm{~km}$ (on average) the wind fluctuations lead the pressure and altitude, whereas for larger scales, the pressure fluctuations leads the wind. At the same transition scale, there is a break in the wind spectrum which we argue is caused by the aircraft starting to accurately follow isobars at the larger scales. In comparison, the temperature and humidity have low coherencies and phases and there are no apparent scale breaks, reinforcing the hypothesis that it is the aircraft trajectory that is causally linked to the scale breaks in the wind measurements.
\end{abstract}

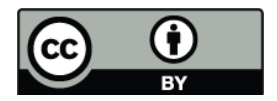

Correspondence to: S. Lovejoy

(lovejoy@physics.mcgill.ca)
Using spectra and structure functions for the wind, we then estimate their exponents $(\beta, H)$ at small $(5 / 3,1 / 3)$ and large scales $(2.4,0.73)$. The latter being very close to those estimated by drop sondes $(2.4,0.75)$ in the vertical direction. In addition, for each leg we estimate the energy flux, the sphero-scale and the critical transition scale. The latter varies quite widely from scales of kilometers to greater than several hundred kilometers. The overall conclusion is that up to the critical scale, the aircraft follows a fractal trajectory which may increase the intermittency of the measurements, but doesn't strongly affect the scaling exponents whereas for scales larger than the critical scale, the aircraft follows isobars whose exponents are different from those along isoheights (and equal to the vertical exponent perpendicular to the isoheights). We bolster this interpretation by considering the absolute slopes $(|\Delta z / \Delta x|)$ of the aircraft as a function of lag $\Delta x$ and of scale invariant lag $\Delta x / \Delta z^{1 / H_{z}}$.

We then revisit four earlier aircraft campaigns including GASP and MOZAIC showing that they all have nearly identical transitions and can thus be easily explained by the proposed combination of altitude/wind in an anisotropic but scaling turbulence. Finally, we argue that this reinterpretation in terms of wide range anisotropic scaling is compatible with atmospheric phenomenology including convection.

\section{Introduction}

Aircraft are commonly used for high resolution studies of the dynamic and thermodynamic atmospheric variables and they are indispensable for understanding the statistical structure of the atmosphere in the horizontal direction. However, aircraft cannot fly in perfect horizontal straight lines, indeed recently (Lovejoy et al., 2004), it was shown that NASA's ER-2

Published by Copernicus Publications on behalf of the European Geosciences Union. 
stratospheric plane can have fractal trajectories. This means that the mean absolute slope increases at smaller and smaller scales being cut off only by the aircraft inertia. For the ER-2, the fractality of the trajectory could be traced to a combination of turbulence and the plane's autopilot which kept the plane near a constant Mach number of 0.7, effectively enforcing long range correlations between the wind and the aircraft altitude.

The interpretation of such data requires assumptions about the turbulence and the mainstream turbulence theories are virtually all isotropic - or at least "quasi isotropic", i.e. with at most "trivial" (scale independent) anisotropies - whereas on the contrary the atmosphere apparently displays "scaling anisotropy". To understand what this means, denote by $\Delta v$ the fluctuation in a turbulent quantity $v$. "Horizontal scaling" means that over a horizontal lag $\Delta x ; \Delta v=\varphi_{h} \Delta x^{H_{h}}$ whereas "vertical scaling" means that over a vertical lag $\Delta z, \Delta v=$ $\varphi_{v} \Delta z^{H_{v}}\left(\varphi_{h}, \varphi_{v}\right.$ are turbulent fluxes, $H_{h}, H_{v}$ are scaling exponents). Strict (statistical) isotropy implies $\varphi_{h}=\varphi_{v}$ and $H_{h}=H_{v}$. However, if $H_{h}=H_{v}$ but $\varphi_{h} \neq \varphi_{v}$ the system is only "quasi-isotropic" or "trivially anisotropic" (structures may be flattened in the vertical but the mean aspect ratio of vertical sections is independent of scale). "Scaling anisotropy" refers to the much stronger (scale by scale, differential) anisotropy which is a consequence of $H_{h} \neq H_{v}$; when $H_{h}<H_{v}$ structures become progressively flatter at larger and larger scales (see Sect. 3 for more details).

If the mainstream theories are correct and $H_{h}=H_{v}=H$, then neither the fractality nor a nonzero aircraft slope is of much consequence for the statistics of the fluctuations, the unique exponent $H$ can be estimated without difficulty. However, if on the contrary the turbulence is anisotropic with different turbulent exponents in the horizontal and vertical directions $\left(H_{h} \neq H_{v}\right)$ then the interpretation may be quite different. Indeed such scaling anisotropy is essentially the mainstream position of the experimentalists who have examined the vertical structure with "Jimspheres", radar, radiosondes or drop sondes (Adelfang, 1971; Endlich et al., 1969; Van Zandt, 1982; Fritts and Chou, 1987; Schertzer and Lovejoy, 1985b; Dewan and Good, 1986; Dewan, 1997; Lazarev et al., 1994; Tsuda et al., 1989; Gardner et al., 1995; Lovejoy et al., 2007, 2009c; see the review in Lovejoy et al., 2008; Lilley et al., 2008; Radkevitch et al., 2008). For example, in the ER-2 case, the fractality of the trajectories leads to anomalous turbulent exponents while the existence of small nonzero slopes can lead to spurious transitions from the true horizontal exponents $\left(H_{h}\right)$ at small scales to the different vertical exponent $\left(H_{v}\right)$ at large horizontal scales with the two separated by a spurious scale break. Lilley et al. (2008) re-examined two of the best known experimental estimates of horizontal wind spectra - the GASP and MOZAIC experiments (Nastrom and Gage, 1983, 1985; Nastrom et al., 1984; Gage and Nastrom, 1986; Lindborg, 1999; Lindborg and Cho, 2001) - and showed that they can readily be explained - i.e. both their small and large scale regimes - by the single wide range scaling 23/9 D anisotropic turbulence predicted by Schertzer and Lovejoy (1985b). Below (Sect. 5) we extend this reevaluation of past measurement campaigns to include those of Gao and Meriwether (1998) (at $6 \mathrm{~km}$ ) and Bacmeister et al. (1996) (stratosphere, 73 ER-2 flights) and show that they also readily fit into this anisotropic but scaling framework.

Today, the use of state-of-the-art high resolution lidar (Lilley et al., 2004) and drop sondes (Lovejoy et al., 2007), has all but proved that the vertical is scaling but with nonstandard exponents. The latter paper is particularly relevant here because it used drop sondes dropped by a Gulfstream 4 aircraft during the month-long Winter Storm 2004 experiment whose simultaneous horizontal aircraft legs are analyzed below. Using 237 drop sondes at roughly $5 \mathrm{~m}$ resolution in the vertical, over 2700 scaling exponents for the horizontal wind were estimated and exponents near the classical values $1 / 3,1$ (the Kolmogorov value and that predicted by quasilinear gravity wave theories respectively, see below) were only obtained in half a dozen cases, with the mean slowly increasing from the (Bolgiano-Obukhov) value 3/5 near the surface to $\approx 0.75$ at higher altitudes. Similarly, Lovejoy et al. (2009c) and Hovde et al. (2009) used the same sondes to determine the corresponding vertical exponents for temperature, pressure, humidity, potential temperature, equivalent potential temperature and air density showing that none had the exponents predicted by classical isotropic theories of turbulence. Note that here and below, reference to Kolmogorov and Bolgiano-Obukhov exponents in no way implies that the original Kolmogorov or Bolgiano-Obukhov isotropic theories are valid; at best, only that their anisotropic generalizations may be (see Schertzer and Lovejoy, 1985a and Tuck, 2008 for a discussion of anisotropic turbulence in the context of fluid mechanics).

If - as these studies suggest - the turbulence really is anisotropic with different horizontal and vertical exponents, then one must find new ways to interpret the aircraft measurements and to estimate the true statistics and horizontal exponents. While this was partially accomplished in the Lovejoy et al. (2004) study of the special ER-2 stratospheric aircraft, it is important to generalize the results and test them on the somewhat different tropospheric aircraft data which attempt to follow isobars rather than isomachs (surfaces of constant Mach number). The goal of this paper is therefore to argue that data from isobaric aircraft flights - especially of the horizontal wind - need to be reinterpreted as a consequence of anisotropic scaling combined with wind/aircraft interactions. In a new paper, Lovejoy et al. (2009b), we attempt to go beyond this to exploit this new interpretation to quantitatively show how the statistics of the basic wind, temperature and humidity fields can be explained by planetary scale multiplicative cascade processes and we estimate the corresponding exponents.

This paper is structured as follows: in Sect. 2 we discuss the salient features of the data, in particular the slopes as functions of scale. In Sect. 3, we develop some theory to help 
interpret anisotropic turbulence measurements. In Sect. 4, we apply these to the data leg by leg and develop a new joint $(\Delta x, \Delta z)$ analysis technique, in Sect. 5 we re-examine several past aircraft measurement campaigns and in Sect. 6 we conclude.

\section{The data}

\subsection{The flight legs, the aircraft slopes ("pitch")}

The Winter Storms 2004 mission was planned by NOAA under the aegis of the National Center for Environmental Prediction to investigate the genesis of storms in the Pacific impacting the west coast of North America. It did so by using targeted observations: the day's flight plan for the Gulfstream G4 aircraft was constructed on the basis of the operational forecast, to drop sondes in the areas that would yield the greatest improvement to the eventual forecast and analysis of the storm's evolution. Given the modus operandi in the area of operation in the first three months of the year, one would expect to encounter cyclonic conditions and jet streams frequently. The scaling behaviour, the wind shears and jet stream characteristics on such targeted flights were indistinguishable from those on the three ferry flights HonoluluAnchorage-Long Beach-Honolulu. More detailed information can be found in Hovde et al. (2009), in Ray et al. (2004) and in Koch et al. (2005).

The Winter Storms 2004 data analysed here involved 10 aircraft flights over a roughly 2 week period over the northern Pacific each dropping 20-30 drop sondes. The plane flew along either the 162,178 , or $196 \mathrm{mb}$ isobars, to within standard deviations of $\pm 0.11 \mathrm{mb}$ (i.e. the pressure level was $\approx$ constant to within $\pm 0.068 \%$ ), see Fig. 1a. Each had one or more roughly constant straight and constant altitude legs more than four hundred kilometers long between 11.9, $13.7 \mathrm{~km}$ altitude (see Table 1 for details, see Fig. $1 \mathrm{~b}$ for all the trajectories, and $1 \mathrm{c}$ for a blow-up showing the relation of the trajectories and the horizontal wind). The data were sampled every $1 \mathrm{~s}$ and the mean horizontal aircraft speed with respect to the ground was $280 \mathrm{~m} / \mathrm{s}$. In addition, we checked that the standard deviation of the distance covered on the ground between consecutive measurements was $\pm 2 \%$ so that the horizontal velocity was nearly constant (in addition, using interpolation, we repeated the key analyses using the actual ground distance rather than the elapsed time and found only very small differences).

The horizontal and vertical winds for the G4 are calculated by solving for the difference of inertial ground speeds in three-dimensions and the flow angle measurements from various sensors. In the short term the Inertial Navigation System (INS) measurements of ground speed are much less noisy than ground speeds taken from GPS positions. While it is possible to smooth the GPS information to gain smooth ground speeds, that has not been done here; the INS data

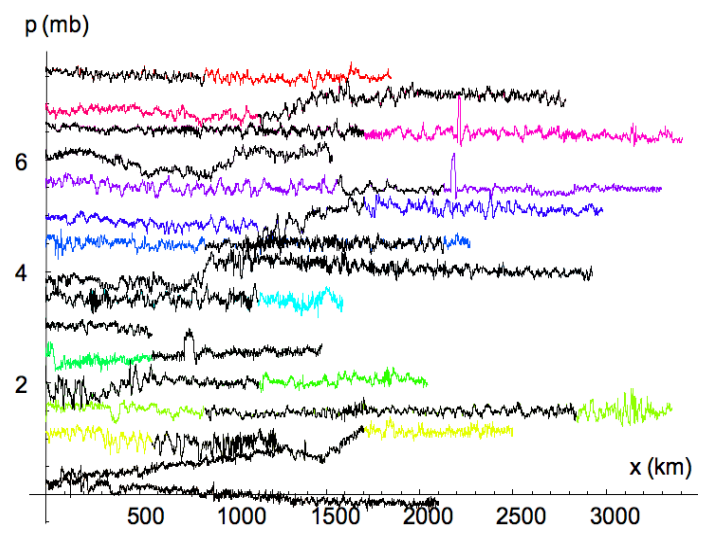

Fig. 1a. The pressure as a function of horizontal position for legs analyzed in this paper. For clarity, the deviations from the minimum pressure are displaced in the vertical by $0.5 \mathrm{mb}$ per curve. The black sections indicate the short legs. The absolute pressures were all between 162 and $196 \mathrm{mb}$.

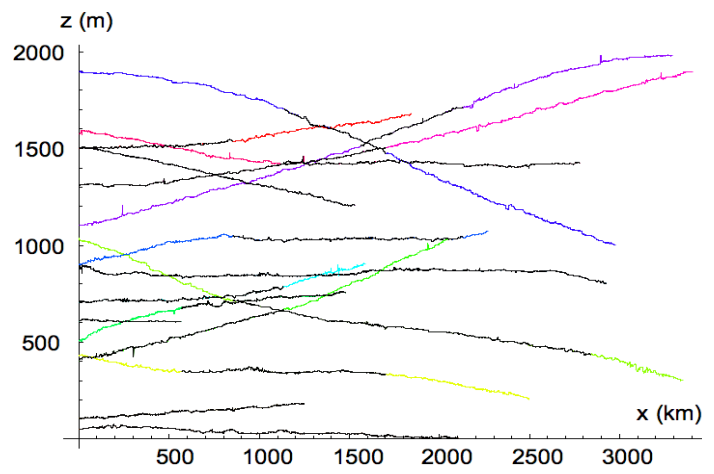

Fig. 1b. The altitude as a function of horizontal position for legs analyzed in this paper. For clarity, the deviations from the minimum altitude are displaced in the vertical by $100 \mathrm{~m}$ per curve. The black sections indicate the short legs.

have been used. It is not clear whether the INS has slower response than the GPS, or whether the latter's high frequency variability is really "noise" or atmospheric variability. There are no corrections of the position of the inertial platform (near the cockpit) to the aircraft centre of gravity (back toward the trailing edge of the wing, probably $10 \mathrm{~m}$ aft of the INS platform).

Since the criterion for a "straight flat leg" was somewhat subjective, we used two different definitions; one which was not so conservative which used 16 straight and flat sections ("legs") constant to within $\pm 450 \mathrm{~m}$ in the altitude and a smaller subset with altitudes to within $\approx \pm 150 \mathrm{~m}$ of a fixed level (see Fig. 1a and $b$ for the distinction). In the end, we did not find significantly different behaviour and the longer legs had the advantage of extending some of our analyses out to distances greater than $3200 \mathrm{~km}$.

As can be seen, in spite of the attempt to use constant altitude legs, it was not quite constant because the pressure levels tended to rise or fall; we see that there is a mean 

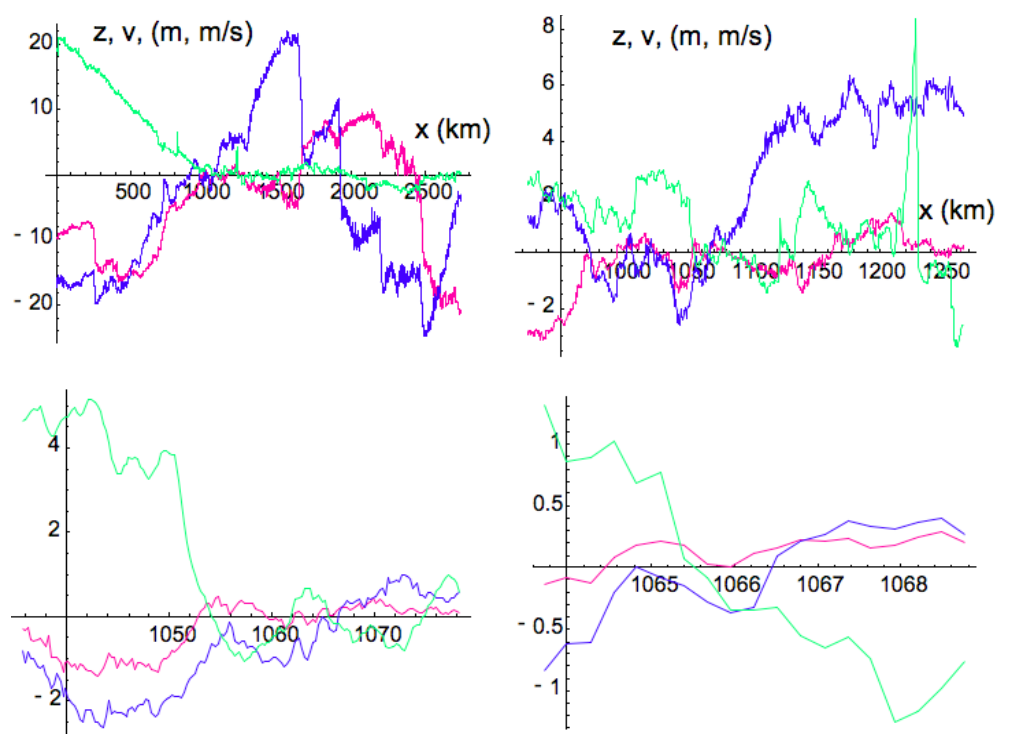

Fig. 1c. This shows four blow ups of factor 8 starting at the upper left, then upper right, lower left, lower right. Green shows the deviations of $\mathrm{z}$ from the $12700 \mathrm{~m}$ of the altitude of the aircraft, (in $\mathrm{m}$ ) but divided by 8, 4, 2, 1 respectively. The red shows the variation in the longitudinal component of the horizontal velocity (in $\mathrm{m} / \mathrm{s}$, deviations from $24.5 \mathrm{~m} / \mathrm{s}$ ), and the blue is the transverse component (in $\mathrm{m} / \mathrm{s}$, deviations from $1.2 \mathrm{~m} / \mathrm{s}$ ). This is for leg 15 , but was typical.

Table 1. This compares the various characteristics of the 16 nearly straight, flat legs considered in this paper. The column $\operatorname{Max}(\Delta z)$ is the difference in altitude between the highest and lowest points on the leg, $\Delta x_{c}$ is the critical scale beyond which the vertical exponent dominates the horizontal (Eq. 9) (here estimated as the geometric mean between the longitudinal and transverse values using regressions on Eq. 11). For legs 2 and 7, the transition was not attained over the entire leg so that only a lower bound is given. We also give the energy flux $\varepsilon$ and the sphero-scale $l_{s}$ are determined by Eq. (12), and the effective dimensionless slope $s_{\text {eff }}$ from Eq. (13). The horizontal shear is the mean for the long legs at horizontal distance half the total distance.

\begin{tabular}{|c|c|c|c|c|c|c|c|c|c|}
\hline $\begin{array}{l}\text { Leg } \\
\text { no. }\end{array}$ & $\begin{array}{c}\text { Short } \\
\text { Length } \\
(\mathrm{km})\end{array}$ & $\begin{array}{c}\text { Short } \\
\text { Max } \\
(\Delta z) \\
(\mathrm{m})\end{array}$ & $\begin{array}{c}\text { Long } \\
\text { Length } \\
(\mathrm{km})\end{array}$ & $\begin{array}{l}\text { Long } \\
\text { Max } \\
(\Delta z) \\
(\mathrm{m})\end{array}$ & $\Delta x_{c}$ & $s_{\text {eff }}$ & $\begin{array}{c}\varepsilon \\
\times 10^{4} \\
\left(\mathrm{~m}^{2}\right. \\
\left.\mathrm{s}^{-3}\right)\end{array}$ & $\begin{array}{l}\text { Shear } \\
\times 10^{6} \\
\left(\mathrm{~s}^{-1}\right)\end{array}$ & (m) \\
\hline 1 & 2100 & 72 & 2100 & 72 & 12.4 & 0.02 & 0.3 & 7.3 & 0.04 \\
\hline 2 & 1248 & 83 & 1248 & 83 & $>1200$ & & 0.2 & 4.9 & \\
\hline 3 & 1136 & 44 & 2496 & 69 & 84 & 0.01 & 40. & 20.4 & 0.14 \\
\hline 4 & 1988 & 285 & 3348 & 737 & 108 & 0.008 & 0.2 & 9.0 & 0.05 \\
\hline 5 & 1136 & 267 & 2044 & 631 & 12.8 & 0.02 & 0.4 & 21.3 & 0.07 \\
\hline 6 & 908 & 89 & 1476 & 260 & 7.6 & 0.02 & 0.2 & 8.1 & 0.03 \\
\hline 7 & 568 & 19 & 568 & 19 & $>400$ & & 0.3 & 20.9 & \\
\hline 8 & 1136 & 84 & 1588 & 206 & 30.4 & 0.01 & 1.2 & 46.4 & 0.08 \\
\hline 9 & 2924 & 100 & 2924 & 100 & 384 & 0.008 & 30. & 6.6 & 0.09 \\
\hline 10 & 1276 & 32 & 2272 & 172 & 40 & 0.02 & 40 . & 7.2 & 0.25 \\
\hline 11 & 568 & 229 & 2980 & 899 & 100 & 0.01 & 0.4 & 10.1 & 0.10 \\
\hline 12 & 568 & 203 & 3292 & 883 & 260 & 0.007 & 0.4 & 8.4 & 0.13 \\
\hline 13 & 1532 & 308 & 1532 & 308 & 52 & 0.02 & 0.2 & 9.9 & 0.60 \\
\hline 14 & 1704 & 204 & 3408 & 597 & 64 & 0.01 & 0.5 & 6.8 & 0.09 \\
\hline 15 & 1648 & 57 & 2780 & 191 & 3.6 & 0.03 & 5. & 8.0 & 0.11 \\
\hline 16 & 852 & 46 & 1844 & 178 & 48 & 0.01 & 2.0 & 5.8 & 0.05 \\
\hline
\end{tabular}

slope ("pitch") of about $0.12 \mathrm{~m} / \mathrm{km}$; this was typical for an entire leg. However, this overall estimate is obviously a very crude characterization; indeed in Lovejoy et al. (2004) it was argued that the ER-2 stratospheric aircraft with special autopilot had a fractal trajectory:

$S_{z}(\Delta x)=\langle|\Delta z(\Delta x)|\rangle \approx a \Delta x^{H_{t r}}$

where $a$ is a constant, $\Delta z(\Delta x)$ is the altitude change over a horizontal lag $\Delta x, "<>$ " indicates ensemble (statistical) averaging and $S_{z}(\Delta x)$ is the (first order) "structure function". For the ER-2 it was found that $H_{t r} \approx 0.55$ with an inner (smoothing) scale of about $3 \mathrm{~km}$ (as a consequence of aircraft inertia smoothing of the otherwise large slope variations) and an outer scale of the fractal regime at about $300 \mathrm{~km}$ due to the slow rise $(\approx 1 \mathrm{~m} / \mathrm{km})$ of the aircraft due to its fuel consumption; the ER-2 roughly followed isomachs rather than isobars. The fractal dimension of the trajectory is $D_{t r}=1+H_{t r}$; for the ER-2, $D_{t r} \approx 1.55$. In order to get a better idea of the typical slopes $(s)$ as functions of scale, for each of the 16 short "legs" we estimated

$\langle|s(\Delta x)|\rangle=\frac{\langle|\Delta z|\rangle}{\Delta x}=S_{z}(\Delta x) / \Delta x=a \Delta x^{H_{s}} ; H_{s}=H_{t r}-1$

these are shown in Fig. 2. From the figure we see that the steepest mean slopes are at the smallest scales and vary from about 0.6 to $1.2 \mathrm{~m} / \mathrm{km}$. It appears that for lags $(\Delta x)$ greater than $\approx 3 \mathrm{~km}$, the slopes follow a suggestive fractal $\Delta x^{H s}$ law with $H_{s}=-2 / 3$ which would result if the vertical displacement was proportional to the fluctuation in the horizontal wind speed, $\Delta z \propto \Delta v$ and if the latter follow a Kolmolgorov law in the horizontal $\langle|\Delta v(\Delta x)|\rangle \approx \varepsilon^{1 / 3} \Delta x^{1 / 3}$ ( $\varepsilon$ is the turbulent energy flux; we confirm this below). Since the lift and drag forces depend on the horizontal wind, a relation of the type $\Delta z \propto \Delta v$ for perturbations is not implausible. If this explanation is correct, the deviations for $\Delta x<3 \mathrm{~km}$ would 


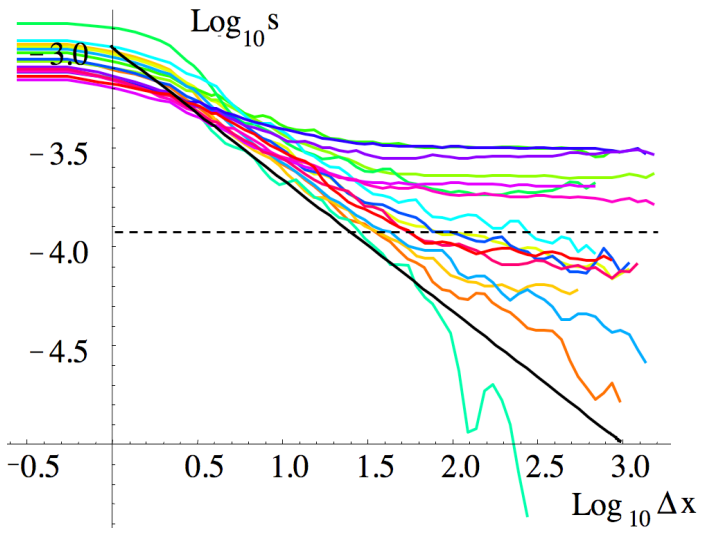

Fig. 2 This shows the mean dimensionless slope $(\langle|s(\Delta x)|\rangle$, Eq. 2) as a function of scale for each of the 16 legs. For reference, we show in black the line $s \approx \Delta x^{-2 / 3}$ corresponding to $H_{t r}=1 / 3$. The structure function $S_{z}$ was estimated by averaging over all disjoint lags. Since the number of such lags decreases with increasing $\Delta x$, the statistics are not so good for the large $\Delta x$; those for lags $>\Delta x_{\max } / 2$ are based on essentially a single atmospheric structure and are not shown. The dashed horizontal line is the mean slope at the half trajectory point (i.e. the mean over all trajectories of $\left.s\left(\Delta x_{\max } / 2\right)=1.2 \times 10^{-4}\right)$.

be the result of aircraft inertia smoothing an otherwise even "rougher" trajectory. At large enough lags in Fig. 2 we see that each trajectory tends to a roughly constant mean absolute slope, although the lag and slope at which this occurs varies greatly from one trajectory to another from about $8 \mathrm{~km}$ to in some cases - greater than the maximum i.e. $>2000 \mathrm{~km}$. These large $\Delta x$, "asymptotic" mean slopes - when they are attained - vary from about $0.3 \mathrm{~m} / \mathrm{km}$ to $<0.1 \mathrm{~m} / \mathrm{km}$; these are roughly constant mean absolute slopes and reflect the large scale slopes of the isobars. The sequence of blowups in Fig. 1c shows that there is indeed some visual evidence for altitude/velocity correlations, particularly with the longitudinal component of the wind although it is subtle; see below. The situation is therefore somewhat different from that of the ER-2 trajectories, being controlled by the isobars rather than the isomachs.

\subsection{Spectral analysis}

To corroborate this interpretation further, we refer the reader to Fig. 3a which shows the spectra of the altitude $z$ for each long leg. For clarity, the spectra are displaced in the vertical and have been normalized or "compensated" by dividing by the theoretical Kolmogorov spectrum $\left(k^{-5 / 3}\right)$. Flat regions thus have spectra $\approx k^{-5 / 3}$. In addition, in order to show the behaviour more clearly - with the exception of the lowest 10 wavenumbers - we have averaged the spectrum over logarithmically spaced bins, 10 per order of magnitude (we also used a standard Hanning window). It can be seen that at $k>(3 \mathrm{~km})^{-1}$ that the spectrum is particularly steep corresponding to smooth behaviour (presumably due to the

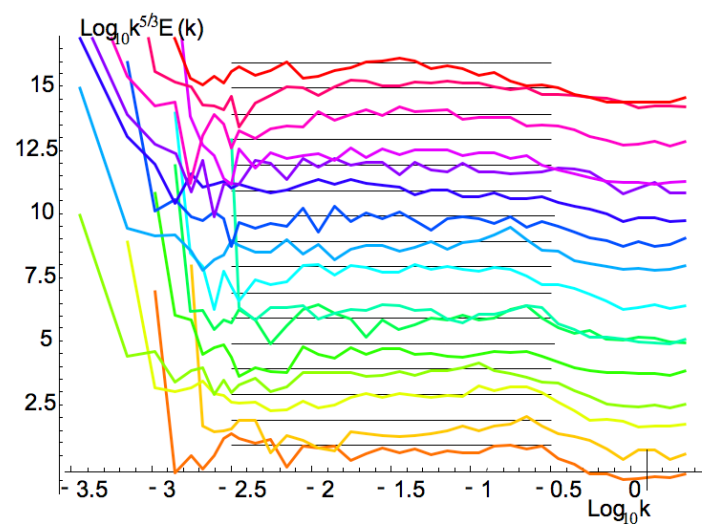

Fig. 3a. The horizontal spectrum of the altitude for each of the legs (1-16 bottom to top, each displaced by an order of magnitude for clarity). In order to see the trends more clearly, for $k>10$, the spectra were averaged over 10 bins per order of magnitude in wavenumber. The spectra are compensated by dividing by $k^{-5 / 3}$ so that the flat regions follow a Kolmogorov $k^{-5 / 3}$ law corresponding to a $\Delta x^{-2 / 3}$ law for the slope in Fig. 2. The Kolmogorov law is found to hold well except at the lowest wavenumbers. The units of the wavenumbers are $\left(\mathrm{km}^{-1}\right)$ the highest wavenumber corresponds to 2 samples, i.e. $2 \mathrm{~s}$ or $560 \mathrm{~m}$.

aircraft inertia as discussed above) whereas in the range of roughly $(4 \mathrm{~km})^{-1}$ to $(100 \mathrm{~km})^{-1}$, the spectrum is $\approx k^{-5 / 3}$. At larger scales $(k<\approx 100 \mathrm{~km})^{-1}$ it rises steeply corresponding to the constant mean slope regime of Fig. 2.

In order to study the relation of this with the measured wind, it is useful to separate the latter into longitudinal and transverse components. This is done because on the one hand, even in isotropic turbulence the latter are in principle different, and on the other hand because we expect that the longitudinal and transverse winds will have somewhat different effects (and hence relationships) on the aircraft altitude (this was indeed found to be the case for the ER-2 measurements). In Fig. $3 \mathrm{~b}$ and $\mathrm{c}$ we show the corresponding compensated spectra for each of the legs. By comparing Fig. 3a, $\mathrm{b}$, and $\mathrm{c}$ we are struck by the fact that they all share the same structure of three regimes at roughly $k=(3 \mathrm{~km})^{-1}$ with the second regime starting at much larger and highly variable scales (investigated in detail below). Since the breaks in the wind spectrum occur where the relation between the spectrum of aircraft altitude changes, this suggests that the vertical aircraft fluctuations strongly influence the measurements over wide ranges.

To clarify the picture, we averaged over the different legs, Fig. 3d. In order to have a uniformly sampled ensemble over the whole range of wavenumbers, we took all the available disjoint 4000 point $(\approx 1120 \mathrm{~km})$ sections (this excluded leg 7 , there were a total of 24 such flight segments in the remaining legs). We see that while the altitude spectrum has a rather accurate $k^{-5 / 3}$ regime over the range roughly $(3 \mathrm{~km})^{-1}$ to $\approx(200 \mathrm{~km})^{-1}$, that in fact the mean wind spectrum has two 


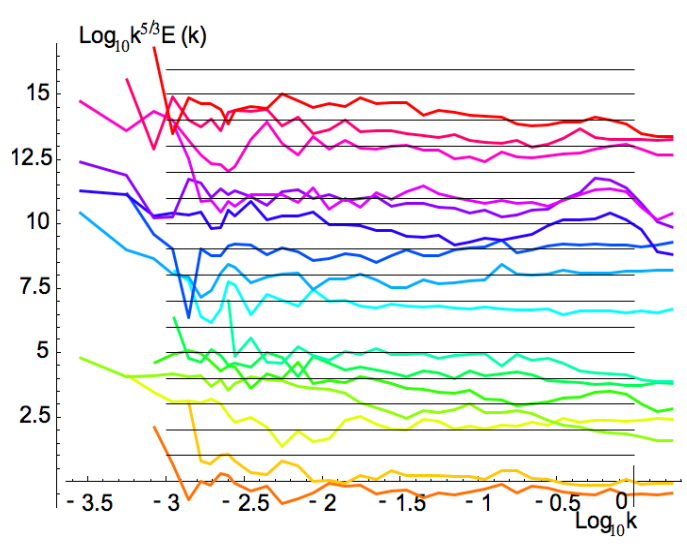

Fig. 3b. The same as Fig. 3a except for the longitudinal component of the wind. The low wavenumber rise seen in most of these corresponds to a roughly $k^{-2.4}$ spectrum; the spectral counterpart of $H_{h}=0.75$ behaviour (with small intermittency corrections).

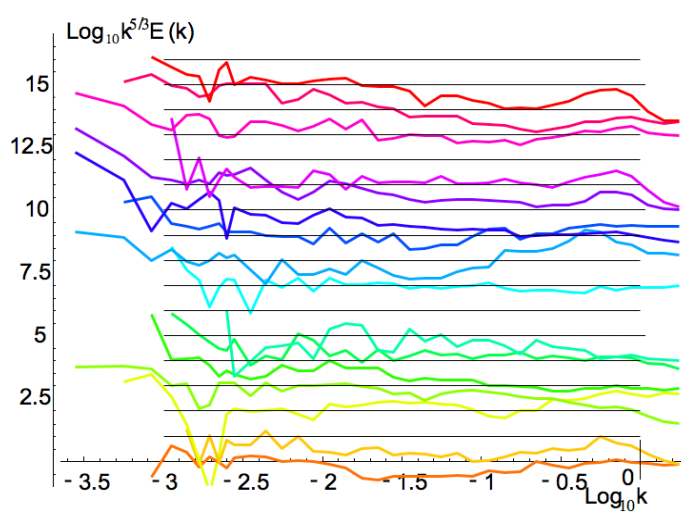

Fig. 3c. The same as Fig. 3b except for the transverse component of the wind.

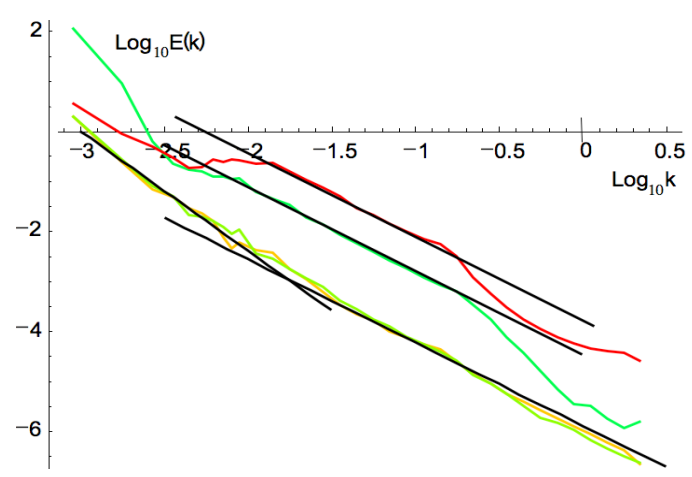

Fig. 3d. The first 4000 points $(1120 \mathrm{~km})$ of the legs (excluding number 7 which was too short) were used to estimate these ensemble spectra which were averaged over all the legs and over, ten wavenumber bins per order of magnitude ( $k$ in units of $\mathrm{km}^{-1}$ ). The pressure (red, top) and altitude (green, second from top), the transverse (orange, bottom) and longitudinal (green, bottom) winds are shown with reference lines indicating the theoretical vertical spectrum $\left(k^{-2.4}\right)$ and the theoretical horizontal spectrum $k^{-5 / 3}$. The average transition wavenumber is about $(40 \mathrm{~km})^{-1}$. regimes, one for $k>\approx(40 \mathrm{~km})^{-1}$ and a $k^{-2.4}$ regime for $k \approx<(40 \mathrm{~km})^{-1}$. Finally, we also show the pressure spectrum finding that it has the same basic regimes as the altitude, and that for $k<\approx(100 \mathrm{~km})^{-1}$ it becomes much less steep while the altitude spectrum is on the contrary steeper suggesting that the aircraft more accurately follows the isobars at these low wavenumbers than at higher ones. We argue below that most individual legs have these transitions although the transition point varies widely from leg to leg. Due to the clear dynamical relation between the wind field and the aircraft trajectory, we should not be surprised at finding a relation between the two. It is therefore of interest to compare this behaviour with that of the (relative) humidity and temperature neither of which are directly linked with the aircraft dynamics. Figure 3e shows the result for the averaged and compensated spectra (with the compensated versions of Fig. 3d for comparison). We see that both have excellent scaling, they are apparently unaffected by the trajectory fluctuations; we examine this more closely below.

\subsection{Cospectral analysis}

In order to further understand the statistical relation between the aircraft altitude and the wind statistics, we can calculate the spectral coherence. Consider the cross-spectrum $S_{h g}$ and normalized (complex) cross-spectrum $\sigma_{h g}$ of two (1-D) functions $h, g$ :

$\sigma_{h g}=\frac{S_{h g}}{\left(S_{g g} S_{h h}\right)^{1 / 2}} ; S_{h g}=\left\langle\tilde{h}(k) \tilde{g}^{*}(k)\right\rangle$

$\tilde{h}(k)=\int_{-\infty}^{\infty} e^{\mathrm{ikx}} h(x) d x ; \tilde{g}(k)=\int_{-\infty}^{\infty} e^{\mathrm{ikx}} g(x) d x$

where " $<$. $>$ " means the "ensemble" (statistical) average, here estimated from the 24 disjoint 4000 point legs and the tilda indicates fourier transform, $k$ is a wavenumber and the asterix means complex conjugate. We can define the coherence $C_{h g}$ and the phase $\theta_{h g}$ as the modulus and phase of $\sigma_{h g}$ :

$\sigma_{h g}(k)=C_{h g}(k) e^{i \theta_{h g}(k)}$

(see e.g. Landahl and Mollo-Christensen 1986). In Fig. 3f, averaging over all the legs, we show these for $h=$ the altitude and with $g$ alternately taken as the longitudinal and transverse wind (left column) and $h$ taken as the pressure and with $g$ alternately taken as the longitudinal and transverse wind (right column). Recall that due to the normalization $0 \leq C \leq 1 ; C$ is a kind of wavenumber by wavenumber correlation coefficient with the important difference that it is positive definite. For identical functions $(h=g), C=1$ while for statistically independent functions, $C(k) \approx 1 / \sqrt{n}$ where $n$ is the number of independent samples used to estimate the ensemble average. Here we considered the first 4000 points of each sufficiently long leg (so that $n=24$ ) hence the coherency for statistically independent wind and altitudes 
is $C(k) \approx 0.20$. In order to estimate the typical deviations around this mean value, we randomly paired altitudes of the $n$-th leg with winds from a randomly chosen but different leg and calculated the resulting $C$ (see Fig. 3f). We notice that the mean of this randomized coherency is near the theoretical value 0.20 , with the "spread" decreasing with wavenumber (due to the fact that the number of wavenumber averaging bins increases with $k$ ).

The coherency is only the modulus; we therefore also considered the phases: $\theta=\theta_{z v}, \theta_{p v}$ (i.e. with $h=z$ and $p$ respectively and $g=v$ in Eq. 3, see Fig. 3g). With this choice, $\theta>0$ indicates that the altitude (pressure) fluctuations lag behind the wind fluctuations while $\theta<0$ indicates the converse. From Fig. $3 \mathrm{f}$ and $\mathrm{g}$ we consider the various regimes.

i) $k>(3 \mathrm{~km})^{-1} ; \log _{10} k>-0.5$

Starting the analysis at the small scales (large wavenumbers), we see that - as expected - due to the inertia of the aircraft which prevents it from rapidly responding to changes in wind, the coherency and phase with respect to the altitude is not statistically signficiant (left column). The situation is more interesting for the pressure (right column) where we see that the transverse component with respect to the pressure is significant, and that the phase of the pressure lags behind the wind fluctuations. This is presumably the effect of fluctuations in the "dynamical pressure" $\Delta p \propto \Delta v^{2}$ caused by the wind changes.

ii) $(40 \mathrm{~km})^{-1}<\boldsymbol{k}<(3 \mathrm{~km})^{-1}$; $\left(-1.5<\log _{10} k<-0.5\right)$

Moving to lower wavenumbers; we first remark that for the longitudinal component, there are apparent significant and even very strong coherencies and phase relations for essentially all the larger scales (although the statistics are poor below about about $\left.k<(500 \mathrm{~km})^{-1}\right)$ with the relation between pressure and wind a bit stronger than that between altitude and wind. Over this range, the transverse component has only small coherencies and phase shifts, being significant only out to about $k<(40 \mathrm{~km})^{-1}$. When we consider the phases, we see that whereas the pressure continues to lag behind the wind $\left(\theta_{p v}>0\right)$, the wind lags behind the altitude changes $\left(\theta_{z v}<0\right)$. This could be a consequence of the autopilot (on a time scale of 10-100 s) adjusting the level due to the smaller scale turbulent trajectory fluctuations (typical time constants for aircraft roll modes are of the order of several seconds and response to rudder and aileron commands are also of this order). Since the aircraft did not fly in any special direction with respect to the wind, the fact that there is such a difference between the longitudinal and transverse components is in itself strong evidence that the aircraft trajectory strongly affects the measurements.

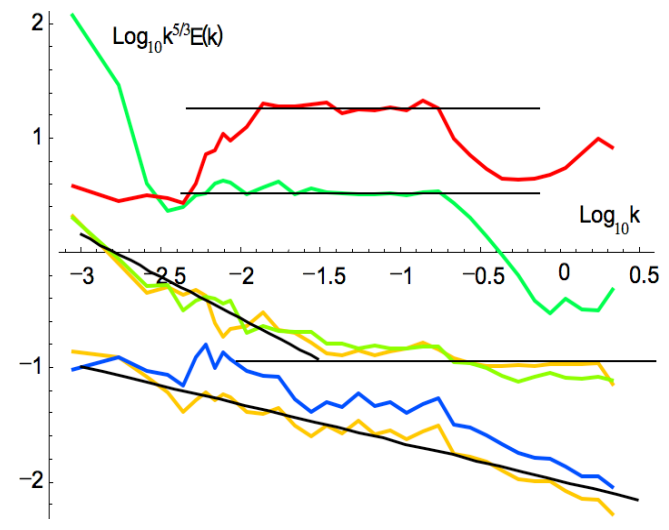

Fig. 3e. Top to bottom: the compensated pressure (red), altitude (green), longitudinal, (green, third from top), transverse, (orange, fourth from top) humidity (blue, second from the bottom) and temperature (orange, bottom). Reference slopes correspond to $k^{-5 / 3}$ (flat), $k^{-2.4}$ and $k^{-2}$. The spectra are for 24 legs each $1120 \mathrm{~km}$ long, averaged over 10 per order of magnitude.

iii) $k<(40 \mathrm{~km})^{-1}$; $\left(\log _{10} k<-1.5\right)$

Finally, at the larger scales where the pressure and then the altitude no longer follow $k^{-5 / 3}$ spectra (Fig. 3e), we see that the phases of both the altitude and pressure with respect to the longitudinal component reverse sign. In this regime, the pressure leads the wind fluctuations while the altitude lags behind. This is presumably the regime in which the aircraft closely follows the isobars. (There is also apparently a tendency for the transverse component to lead the pressure but the corresponding coherence is not statistically significant). From Fig. 3d and e we see that this is also the regime where the wind spectrum follows the $k^{-2.4}$ rather than $k^{-5 / 3}$ law; below we argue that it is this "imposed" vertical displacement that leads to the spurious appearance of the vertical exponent 2.4. This regime is consistent with the aircraft closely following isobars with the latter causing the wind and altitude fluctuations.

Large scale pressure/wind relations could arise naturally in the following way: along an isobar we have $d z=$ $-\frac{\partial p}{\partial x} / \frac{\partial p}{\partial z} d x \approx d x \frac{\partial p}{\partial x} /(\rho g)$ where $x$ is a coordinate parallel to the aircraft trajectory and we have used the hydrostatic approximation. If we also make the geostrophic approximation, $(\partial p / \partial x) /(\rho g) \approx-f v_{y}$, then we obtain the "geostrophic" slope: $s_{\text {geo }}=d z / d x \approx-f v_{y} / g$ where $v_{y}$ is a transverse wind component and $f$ is the Coriolis parameter.

Using data from the legs averaged at $40 \mathrm{~km}$ from the aircraft campaign we found that the actual slopes were only a little larger than these "geostrophic" slopes with mean ratio: $2.2 \pm 1.4$. This gives evidence that the slopes of the isobars are indeed linked to the wind at these scales. This long-range meteorological effect could lead to large vertical fluctuations so that the wind fluctuations are mainly due to the vertical displacement of the aircraft along isobars. We could note that 

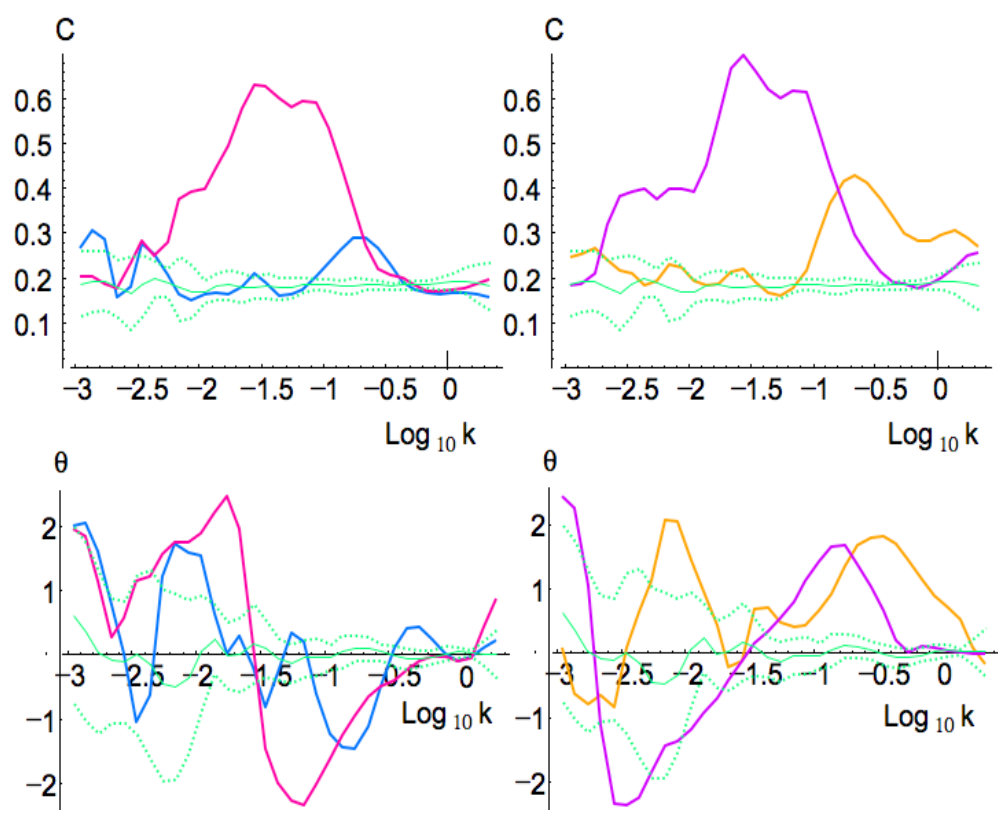

Fig. 3f. This shows the coherency (top row) and phases (bottom row) of the cross spectra of altitude (left column) and pressure (right column) as function of horizontal wavenumber $k$, units $\mathrm{km}^{-1}$. The transverse wind is blue (left), orange (right), the longitudinal wind is red (left), purple (right). The coherency and phases were averaged over logarithmically spaced bins, 10 per order of magnitude (except for the lowest 10 wavenumbers). Green shows the mean (thick) and standard deviation (dashed) of the randomized coherencies and phases as discussed in the text. Phases and coherencies are only statistically significant when outside the corresponding ranges.
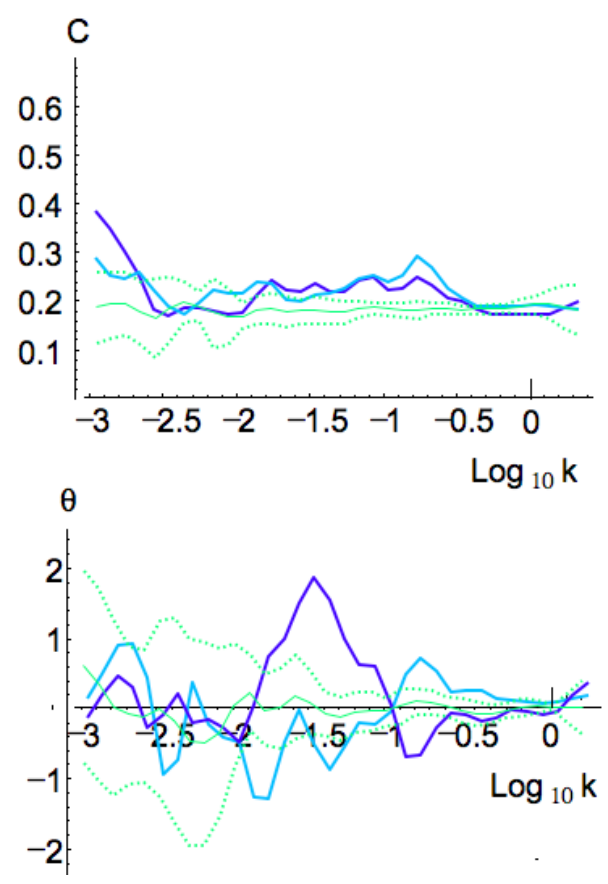

Fig. 3g. Similar to Fig. 3f, this shows the coherencies (top) and the phases (bottom) of the cross spectra of altitude with temperature (blue), humidity (cyan), $k$ in units $\mathrm{km}^{-1}$.
Fig. 2 already shows that the scale $40 \mathrm{~km}$ is only an average which hides very large leg to leg variations; this is further confirmed in Fig. 5 and in the sections below.

In Fig. $3 \mathrm{c}$ we see that $k \approx(40 \mathrm{~km})^{-1}$ is indeed the critical scale for the (average) wind spectrum; for $k>(40 \mathrm{~km})^{-1}$ the vertical fluctuations are not dominant and the spectrum is the (apparently unbiased) horizontal Kolmolgorov value 5/3 where as for $k<(40 \mathrm{~km})^{-1}$ the vertical fluctuations are sufficiently large so that the vertical exponent 2.4 is obtained.

As a final check, we also considered the temperature and humidity coherencies and phases (Fig. 3g). We see that over the regime $(40 \mathrm{~km})^{-1}<k<(3 \mathrm{~km})^{-1}$ there are only low coherencies and small phases for both, becoming insignificant for $k<(100 \mathrm{~km})^{-1}$. The most statistically significant - the temperature phases - indicate that there is a lag with respect to the altitude, as expected if the altitude fluctuations were imposed by the autopilot. The overall weak link between the trajectory statistics and the temperature and humidity fluctuations is consistent with the excellent spectral scaling $k^{-\beta}$ (with $\beta=2.13,2.10$, respectively) over the entire range.

Finally, in a new paper (Lovejoy et al., 2009b) we take a closer look at the scale by scale behaviour of the statistics isolating the contribution of turbulent intermittency to the overall variability. This analysis shows that the aircraft altitude is extremely intermittent for scales smaller than $\approx 40 \mathrm{~km}$ but is much less so for the larger scales. This reinforces the picture developed above: the turbulence strongly affects the measurements at the smaller scales whereas at the larger scales, 
the aircraft closely follows the (relatively smooth) isobars. According to this interpretation, the small scale fractality of the trajectories - while possibly increasing the intermittency - does not seriously bias the small scale wind spectrum which approximates that of an isoheight. However, at the larger scales, we obtain the different isobaric spectrum (which below we argue is the same as the vertical spectrum).

\section{Understanding the effects of vertical aircraft motion on the velocity fluctuations}

Let us consider a fairly general case of anisotropic but scaling turbulence so that the fluctuations in the horizontal velocity over a horizontal lag $\Delta x$ and vertical lag $\Delta z$ follow:

$\Delta v=\varphi_{h} \Delta x^{H_{h}} ; \Delta v=\varphi_{v} \Delta z^{H_{v}}$

where $\varphi_{h}, \varphi_{v}$ are the turbulent fluxes dominant in the horizontal and vertical directions respectively and $H_{h}, H_{v}$ are the corresponding exponents. The (isotropic) Kolmogorov law is recovered with $\varphi_{h}=\varphi_{v}=\varepsilon^{1 / 3}, H_{h}=H_{v}=1 / 3$ where $\varepsilon$ is the energy flux. In comparison, the original 23/9 D model of anisotropic scaling turbulence (Schertzer and Lovejoy, 1985 b) in which the horizontal is dominated by the energy flux $\left(\varepsilon, \mathrm{m}^{2} \mathrm{~s}^{-3}\right)$ and the vertical by buoyancy variance flux $\left(\phi, \mathrm{m}^{2} \mathrm{~s}^{-5}\right)$ is obtained with $\varphi_{h}=\varepsilon^{1 / 3}, \varphi_{v}=\phi^{1 / 5}, H_{h}=1 / 3$, $H_{v}=3 / 5$. Similarly, the popular quasi-linear gravity wave models (Dewan and Good, 1986; Dewan, 1997; Gardner, 1994; Gardner et al., 1993) typically take $\varphi_{h}=\varepsilon^{1 / 3}, \varphi_{v}=N$ (the Brunt Väisälä frequency; this is not a turbulent flux, a fact which is a serious weakness of that theory) so that $H_{h}=1 / 3, H_{v}=1$. (Interestingly, Van Zandt, 1982 was a forerunner of these quasi-linear gravity wave theories and proposed - purely empirically - a vertical spectral exponent of -2.4 , in accord with our results below). In order to write these anisotropic models in a form valid for any vector in the vertical plane $\Delta r=(\Delta x, \Delta z)$ we can use the formalism of Generalized Scale Invariance (Schertzer and Lovejoy, 1985a) and write:

$\Delta v=\varphi_{h}\|\underline{\Delta r}\|^{H_{h}}$

where the scale function (indicated by the double bars) replaces the usual vector norm appropriate for isotropic turbulence:

$$
\begin{aligned}
& \|\underline{\Delta r}\|=l_{s}\left(\left(\frac{\Delta x}{l_{s}}\right)^{2}+\left(\frac{\Delta z}{l_{s}}\right)^{2 / H_{z}}\right)^{1 / 2} ; \\
& H_{z}=\frac{H_{h}}{H_{v}} ; l_{s}=\left(\frac{\varphi_{h}}{\varphi_{v}}\right)^{1 /\left(H_{v}-H_{h}\right)}
\end{aligned}
$$

where $H_{z}$ is the exponent characterizing the degree of stratification $\left(H_{z}=1\right.$ corresponds to isotropic 3 -D turbulence, $H_{z}=0$ to isotropic 2-D turbulence) and $l_{s}$ is the "sphero-scale" socalled because the structures are roundish at that scale. The scale function need only satisfy a fairly general scale equation, so that the above form is only the simplest "canonical" scale function but is adequate for our purposes. It can be verified that if we successively take $\underline{\Delta r}=(\Delta x, 0)$ and $\underline{\Delta r}=(0, \Delta z)$ that we recover Eq. (5).

In such a turbulence, the volumes of structures (assumed isotropic in the horizontal) change with horizontal scale $\Delta x$ as $\Delta x^{D_{e l}}$ with $D_{e l}=2+H_{z}$. The 23/9 D model derives its name because $H_{z}=(1 / 3) /(3 / 5)=5 / 9$; the quasi-linear gravity wave model has $H_{z}=1 / 3$ and therefore $D_{e l}=7 / 3$ and we have noted that the classical 2-D and 3-D isotropic turbulences have $H_{z}=0,1$ hence $D_{e l}=2,3$ respectively. The 23/9 D model of stratification was found to be obeyed quite precisely for passive scalar densities estimated by lidar (i.e. with the above scale function replacing the vector norm in the isotropic Corrsin-Obukhov law of passive scalar advection (Lilley et al., 2004, 2008); cf. $H_{z}=0.55 \pm 0.02$ ). Using drop sondes, it was also found for the lower $2 \mathrm{~km}$ or so of the atmosphere that the horizontal velocity (Lovejoy et al., 2007), also had $H_{z} \approx 0.55$. However small but significant deviations were observed for higher altitudes so that $H_{v} \approx 0.75$ and hence (assuming $H_{h}=1 / 3$ ) we infer that $H_{z} \approx 0.44$. The origin of these deviations for the horizontal wind from the theoretical value is still not understood, they are especially puzzling since the theory apparently holds quite accurately for passive scalars.

In order to understand the effect of the vertical trajectory variability on the horizontal wind statistics, consider a section with constant slope $s$ so that $\Delta z=s \Delta x$ :

$\Delta v=\varphi_{h} l_{s}^{H_{h}}\left(\left(\frac{\Delta x}{l_{s}}\right)^{2}+\left(\frac{s \Delta x}{l_{s}}\right)^{2 / H_{z}}\right)^{H_{h} / 2}$

When considering the ER-2 trajectory, (Lovejoy et al., 2004) pointed out that if $s$ was constant, then there would exist a critical lag $\Delta x_{c}=l_{s} s^{1 /\left(H_{z}-1\right)}$ such that for $\Delta x>\Delta x_{c}$, the second term would dominate the first and we would obtain:

$$
\begin{aligned}
& \Delta v=\varphi_{h} \Delta x^{H_{h}} ; \Delta x \ll \Delta x_{c} \\
& \Delta v=\varphi_{v} s^{H_{v}} \Delta x^{H_{v}} ; \Delta x \gg \Delta x_{c}
\end{aligned}
$$

We would therefore expect a spurious break in the horizontal scaling at $\Delta x_{c}$ after which the aircraft would measure the vertical rather than horizontal statistics with exponent $H_{v}$ rather than $H_{h}$. In the case of the ER-2, this was indeed the case for the longest lags dominated by the constant slope regime at around $300 \mathrm{~km}(s \approx 1 \mathrm{~m} / \mathrm{km}$ caused by the aircraft losing weight due its fuel consumption). However, in their reinterpretation of the classical tropospheric turbulence campaigns using commercial airplanes (GASP, MOZAIC), Lilley et al., 2008 , found that the horizontal wind spectra and structure functions respectively could be explained if there was a transition from horizontal to vertical exponents at the somewhat smaller $\Delta x_{c}$ 's of around 30-50 km (i.e. about the same as the mean found here, Fig. 3c), see also the reanalyses in Sect. 5 of the spectra in Gao and Meriwether (1998), Bacmeister et 
al. (1994), Cho and Lindborg (2001), and Gage and Nastrom (1986). Unlike the ER-2 structure function, in these roughly isobaric trajectories, there was no indication of a significant intermediate fractal dominated regime in which the turbulent exponents are apparently biased by the long range correlation between the aircraft position/altitude and the turbulence it measures.

In order to understand the general effect of a fractal trajectory on the $\Delta v$ statistics, we may consider a fractal trajectory obeying Eq. (1) with the simplifying "mean field" hypothesis that the mean result Eq. (1) can be used in place of $\Delta z$ in Eq. (7) (this is equivalent to ignoring the correlations between the trajectory and the horizontal wind). It implies:

$\langle|\Delta v|\rangle=\varphi_{h} l_{s}^{H_{h}}\left(\left(\frac{\Delta x}{l_{s}}\right)^{2}+\left(\frac{a}{l_{s}}\right)^{2 / H_{z}}\left(\Delta x^{2}\right)^{H_{t r} / H_{z}}\right)^{H_{h} / 2}$

we therefore see that there exists a critical trajectory exponent $H_{t r c}=H_{z}$ such that for $H_{t r}>H_{t r c}$ a spurious transition will occur at a critical $\Delta x_{c}$ such that the second (vertical) term will dominate at $\Delta x>\Delta x_{c}$, while for small scales $\Delta x<\Delta x_{c}$ the first (horizontal) term will dominate. However, on the contrary for $H_{t r}<H_{t r c}$ we find that the fractal nature of the trajectory will not lead to spurious scaling, that it will not affect the horizontal exponent. We can now understand the key difference between our Gulfstream 4 data and the ER-2. Over the range $\approx 3 \mathrm{~km}<\Delta x<300 \mathrm{~km}$, the latter had an anomalous regime with $H_{t r} \approx 0.55 \approx H_{t r c}$ so that the above "mean field" type argument breaks down; we must carefully consider the (nontrivial) correlations between the wind and the trajectory, they can be important over a wide range. However, from Fig. 2, we can see that the Gulfstream 4 tropospheric isobaric trajectories analyzed here are different; they tend to involve abrupt transitions from $H_{t r} \approx 1 / 3$ to $H_{t r} \approx 1$ (i.e. $H_{s} \approx-2 / 3$ to $H_{s} \approx 0$ ) so that a spurious transition from horizontal to vertical exponents may or may not occur depending on the magnitude of the vertical fluctuations $(a)$, the value of $l_{s}$ (which depends on the relative magnitudes of the horizontal and vertical turbulent fluxes, Eq. 5), and the point at which the transition from $H_{t r} \approx 1 / 3$ to $H_{t r} \approx 1$ occurs.

Since turbulence is highly intermittent, in order to obtain robust estimates of exponents, experimentalists average their velocity fluctuations over as many lags as possible. Since $H_{v}>H_{h}$, it is enough that only some lags have a transition from horizontal to vertical behaviour for the spurious vertical scaling to dominate the ensemble statistics for large enough $\Delta x$. For each leg and for the averages over all the lags $\Delta x$, we therefore anticipate (cf. Eq. 8) that:

$\langle|\Delta v|\rangle=\left((A \Delta x)^{2}+(B \Delta x)^{2 H_{v} / H_{h}}\right)^{H_{h} / 2}$

for some empirically determined constants $A, B$. In order to test the hypothesis and to estimate the key exponents $H_{h}$, $H_{v}$, for each pair $\left(H_{h}, H_{v}\right)$ we performed a regression on $\log _{10}\langle|\Delta \nu|\rangle$ to determine the constants $A, B$ which minimized the root mean square residuals (error). In Fig. 4a

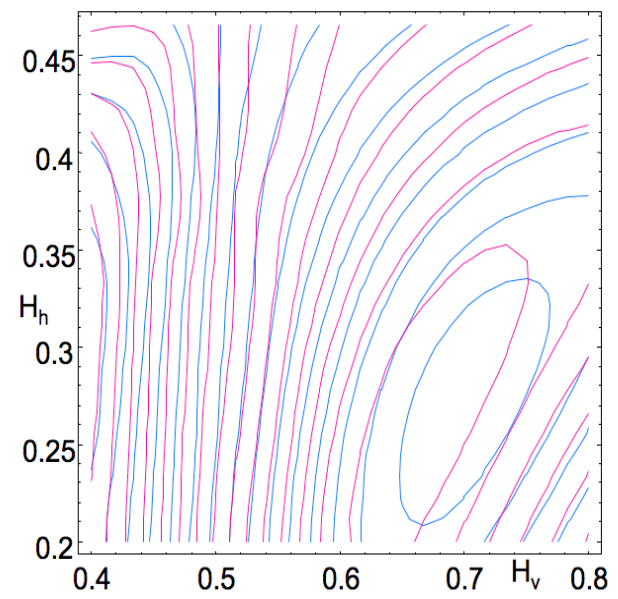

Fig. 4a. A contour plot of the rms errors in estimating $\log _{10}\langle|\Delta v|\rangle$ using the formula Eq. (11). The longitudinal and transverse components of the horizontal winds are shown in pink, blue respectively. The centre of the diagram corresponds to the theoretical values $\left(H_{h}, H_{v}\right)=(1 / 3,3 / 5)$. The minima are at $\left(H_{h}, H_{v}\right)=(0.26 \pm 0.07$, $0.65 \pm 0.04),(0.27 \pm 0.13,0.67 \pm 0.09)$ for the transverse and longitudinal components respectively.

we display a contour plot showing the behaviour of the error for components of the horizontal wind both transverse and longitudinal to the aircraft heading. There is a broad minimum; statistical analysis shows that minimum occurs at $\left(H_{h}, H_{v}\right)=(0.26 \pm 0.07,0.65 \pm 0.04),(0.27 \pm 0.13,0.67 \pm 0.09)$ for the transverse and longitudinal components respectively (the rms error in $\log _{10}\langle|\Delta v|\rangle$ at the minimum was about \pm 0.03 in both cases corresponding to deviations of only 100 $\left(10^{0.03}-1\right) \approx \pm 7 \%$ over $3-4$ orders of magnitude in scale). As discussed earlier, the longitudinal component is more coherent with the altitude, this would explain its slightly larger error. Given the large uncertainties, we can see that the $H_{h}$ estimates are compatible with the Kolmogorov value $H_{h}=1 / 3$ while $H_{v}$ is compatible with both the Bolgiano - Obukhov value $3 / 5$ and the slightly larger value $\approx 0.75$ observed from the (simultaneous) drop sondes in lower $1 \mathrm{~km}$ and the upper $12-13 \mathrm{~km}$ altitude range respectively (corresponding to $H_{z}=5 / 9,0.44$, respectively). Below, we shall see that not all legs show $\Delta x^{H_{v}}$ regimes, and for some it is only visible for the largest $\Delta x$; this effect may lead to an underestimate of $H_{v}$.

\section{Leg by leg and $(\Delta x, \Delta z)$ analyses}

Now that we have reasonable estimates of the exponents and in order to understand the results better - we can again consider the individual legs. Figure $5 \mathrm{a}$ and $\mathrm{b}$ show the individual structure functions for each leg for the longitudinal and transverse components with regressions to the form Eq. (11) constrained to have $H_{h}=1 / 3$ and $H_{v}=3 / 5$ (thin line), $H_{v}=3 / 4$ (thick line). We see that the theoretical fits (with er- 
rors indicated in Fig. $4 b \approx \pm 0.04$ ) are very good for both values of $H_{v}$ with not much difference between them.

Using the regression coefficients $A, B$ obtained with $H_{h}=1 / 3, \quad H_{z}=4 / 9$, we can estimate the critical $\Delta x_{c}$ at which the two terms in Eq. (11) are equal: $\Delta x_{c}=$ $A^{H_{z} /\left(1-H_{z}\right)} / B^{1-H_{z}}$ (Table 1). We see that in two cases legs 2 and 7 - that the $\Delta x^{1 / 3}$ law holds well over the entire leg so that no transition is observed (the corresponding entry is blank). Close examination of the corresponding slopes (Fig. 2) shows that these are cases with particularly long $s(\Delta x) \approx \Delta x^{-2 / 3}$ regimes which - following our preceding analyses - favour the horizontal exponents (see also the spectra in Fig. 3). If in addition the $l_{s}$ value is particularly large - and (Radkevitch et al., 2008) shows empirically that it has huge fluctuations; the probability tail has a "fat" power law fall-off with exponent $\approx 1.33$ - then there will be no transition over the observed range of lags $\Delta x$.

The direct regressions on the structure functions only give the coefficients $A, B$; these cannot be used directly to estimate $l_{s}$ and $\varepsilon$. In addition, the analysis so far cannot rule out the possibility that the large scale turbulence is isotropic with genuine (rather than spurious) horizontal exponent $H_{h} \approx 0.7-$ 0.75 and with highly variable transition point. In this case for $\Delta x \gg \Delta z$, the $\Delta z$ values will be statistically irrelevant for $\Delta v$; only the $\Delta x$ values will be important. In order to rule out the latter possibility and to estimate $l_{s}$ and $\varepsilon$ we must use a different analysis technique. The key is to use the information of the joint $\Delta v$ dependence on both $\Delta x$ and $\Delta z$. Rewriting Eq. (7) we find:

$$
\begin{aligned}
& \Delta=\left(\overline{\varepsilon^{1 / 3}}\right)_{\zeta}\left(1+l_{s}^{1-1 / H_{z}} \zeta^{-1}\right)^{1 / 3} \\
& \Delta=\frac{(\overline{|\Delta v(\Delta x, \Delta z)|})_{\zeta}}{\Delta x^{1 / 3}} ; \zeta=\frac{\Delta x}{\Delta z^{1 / H_{z}}}
\end{aligned}
$$

Where we use the average over constant $\zeta$ (denoted by the overbar and subscript) of $\varepsilon^{1 / 3}$ and the normalized gradient $\Delta . \quad \zeta$ is the "scale invariant lag" since under generalized scale changes $T_{\lambda}=\lambda^{-G}$ (where $G$ is the generator of the scale changing group - in this case the $2 \times 2$ matrix $\mathbf{G}=((1,0),(0$, $\left.\left.H_{z}\right)\right)$ ) if we start with a unit vector $\underline{\Delta r_{1}}=\left(\Delta x_{1}, \Delta z_{1}\right)$ with associated $\zeta_{1}=\Delta x_{1} \Delta z_{1}^{-1 / H_{z}}$, the scale changing operator $T_{\lambda}$ yields the $\lambda$ times smaller reduced vector $\underline{\Delta r_{\lambda}}=T_{\lambda} \underline{\Delta r_{1}}$ but with, $\zeta_{\lambda}=\left(\lambda \Delta x_{1}\right)\left(\lambda^{H_{z}} \Delta z_{1}\right)^{-1 / H_{z}}=\zeta_{1}$, i.e. unchanged. In order to improve the statistics, we used all the $n(n-1) / 2$ pairs of measurements for each $n$ point long leg, hence yielding robust behaviour and parameter estimates. Figure 6 shows the leg by leg result (on the short legs) along with the optimum regression to determine $\varepsilon, l_{s}$ (the former is simply the large $\zeta$ asymptote); these are given in Table 1 . The figure shows that the theoretical form fits very well over an impressive 8 orders of magnitude in $\zeta$. The main deviations are at the small $\zeta$ values, but this reflects that the fact that the small $\zeta$ values are not numerous so that the statistics are not

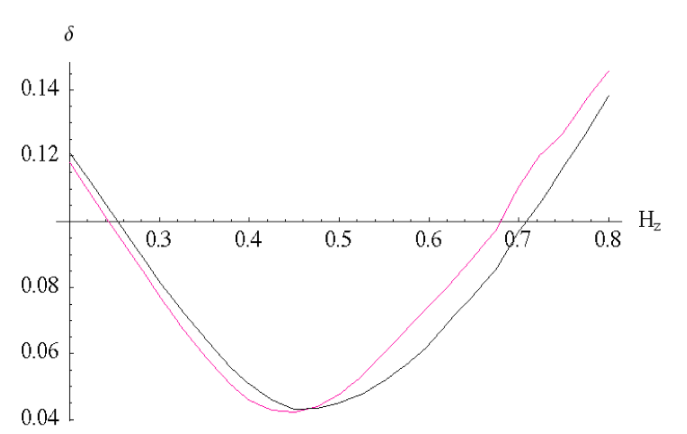

Fig. 4b. The rms error in estimating $\log _{10}\langle|\Delta v|\rangle$ for longitudinal (black) and transverse (red) components respectively obtained by fixing $H_{h}=1 / 3$. The minima correspond to the estimates: $H_{z} \approx 0.46 \pm 0.05,0.45 \pm 0.05$ for longitudinal and transverse components respectively.

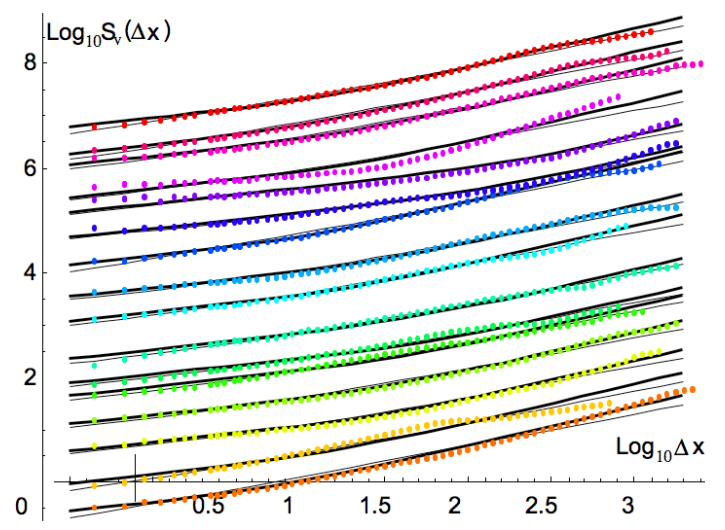

Fig. 5a. This shows the first order structure function for the longitudinal component of the horizontal wind for each of the 16 legs, each displaced by 0.5 in vertical for clarity. $\Delta x$ is the horizontal distance in $\mathrm{km}$. The thin line is the regression to the form Eq. (11) with $H_{z}=4 / 9$ while the thick line has $H_{z}=5 / 9$.

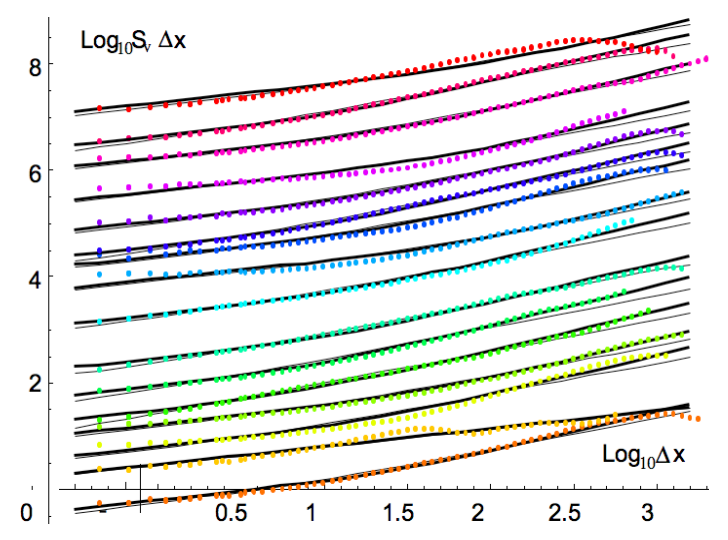

Fig. 5b. Same as Fig. 5a except for the transverse component. 


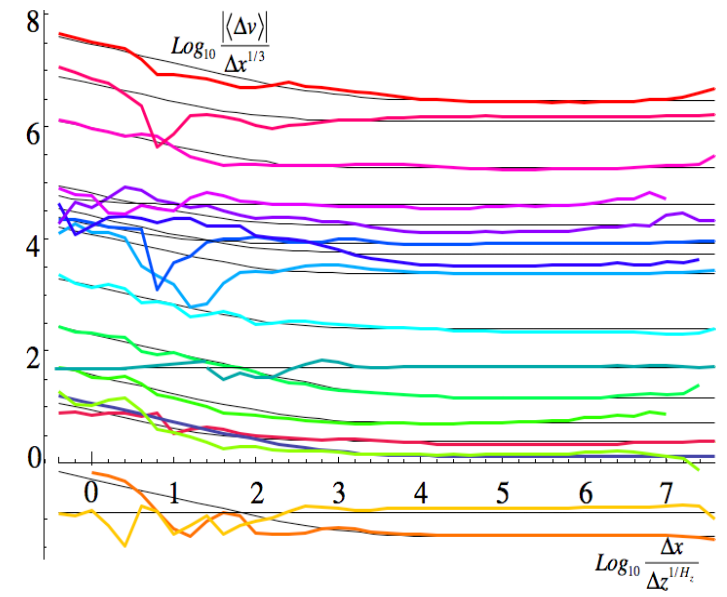

Fig. 6. This shows the normalized mean velocity difference $\Delta$ as a function of the scale invariant lag $\zeta=\Delta x / \Delta z^{1 / H_{z}}$ with $H_{z}=4 / 9$. The legs are shown displaced vertically for clarity (legs $1-16$, bottom to top). The statistics of long flat lags are to the right, short, highly sloped lags to the left. The black lines give regressions to the form Eq. (12). Using $H_{z}=5 / 9$ does not change the appearance very much.

well estimated; they are from rare large vertical "jumps" over short distances. We note that there are no signs of deviations from the theoretical behaviour at large $\zeta$ corresponding to long and flat displacements. In other words, we can rule out a large scale transition to isotropic $H_{h}=H_{v} \approx 0.7$ turbulence since the longest, flattest displacements have $H_{h}=1 / 3$.

Aside from the strong support that the figure gives to our conclusions about the effect of anisotropic turbulence, we can also note that it confirms that legs 2 and 7 have nearly perfect $\Delta x^{1 / 3}$ behaviours over the entire range. We can also note from the table that the values of $\varepsilon$ are highly variable (as expected) but the (geometric) mean $\left(\approx 4 \times 10^{-4} \mathrm{~m}^{2} \mathrm{~s}^{-3}\right)$ is not so far from the "typical values" $10^{-3}-10^{-4} \mathrm{~m}^{2} \mathrm{~s}^{-3}$ measured elsewhere. Also shown in Table 1 is the mean horizontal shear over the legs; it is not obviously related to any of the other characteristics listed.

We see that the estimates of $l_{s}$ are in the range $3 \mathrm{~cm}$ to about $70 \mathrm{~cm}$ which is exactly the range of the direct estimates from lidar in Lilley et al. (2004) (9 cross-sections of passive scalar lidar backscatter ratios, each with $2<l_{s}<80 \mathrm{~cm}$ ) and a little larger than the estimate from the mean ER-2 data $\left(l_{s} \approx 4 \mathrm{~cm}\right)$. Recall that since $H_{v}>H_{h}, l_{s}$ is the scale at which structures begin to become flattened in the horizontal. Using this value of $l_{s}$, we can calculate an "effective slope" $s_{\text {eff }}$ which is the constant slope that would explain the transition at $\Delta x_{c}$ from horizontal to vertical scaling statistics (see Table 1):

$s_{\text {eff }}=\frac{B l_{S}}{\left(A l_{S}\right)^{1 / H_{z}}}$

We see that the values are quite large - in the range 7$25 \mathrm{~m} / \mathrm{km}$; this shows that the behaviour cannot be under- stood in terms of a roughly constant $s$ over an entire leg; the wind/altitude coherency is more subtle than that.

We might mention at this point that here we have considered that the scaling exponents $H_{h}, H_{v}$ apply to the behaviour along orthogonal axes defined by the local gravity field. However, this may be only an approximation: theoretically the axes need not be exactly orthogonal (corresponding to non diagonal generators of the anisotropy). This might arise as a consequence of some strong shear for example. The physically relevant $s$ would be then slope with respect to these axes and not with the local gravity field.

\section{Comparison with other aircraft studies}

We have argued that aircraft measurements of wind have systematically ignored the effect of fluctuations/variations in the altitude of the measurements and that as a consequence, at large enough scales the measured wind fluctuations spuriously have vertical scaling exponents rather than the true horizontal exponents (for the spectra, $\approx 2.4$ and $\approx 5 / 3$, respectively). Equivalently, at small scales, the aircraft follows a fractal trajectory, yet apparently measures isoheight exponents (with perhaps biased - but in any case small - intermittency corrections) whereas at large scales, the aircraft accurately follows isobars with (different) isobaric exponents. Although we mentioned that this hypothesis has been verified in Lilley et al. (2008) on two of the main atmospheric campaigns (GASP and MOZAIC), we would like to revisit these quickly along with two others showing that they are very close to those here (Fig. 3d) and can be convincingly explained by the combination of vertical aircraft fluctuations coupled with anisotropic but scaling turbulence.

The GASP experiment was perhaps the most influential experiment to date on the horizontal spectrum, being generally interpreted as lending support to 2-D turbulence at large scales. However, this interpretation is fraught with difficulties since the claimed 2-D $k^{-3}$ behaviour would only be in the narrow range between about 500 and $3000 \mathrm{~km}$; see Fig. 7a. Restricting ourselves to isotropic turbulences this would imply that the 3-D $k^{-5 / 3}$ range extends way beyond the atmospheric scale height $10 \mathrm{~km}$ requiring that the $3-\mathrm{D}$ turbulence be "squeezed to become nearly two dimensional" (Högstrom et al. 1999) with an energy flux source localized in scale somewhere around $500 \mathrm{~km}$ (Lilley, 1983; Högstrom et al., 1999, proposes that this source might be convection). Additionally, this model would require an enstrophy source at around $3000 \mathrm{~km}$ - given possibly by baroclinic instabilities (see also Lilly, 1989 for more elaboration).

While this classical interpretation is forced - involving as it does two ad hoc sources and an unclear "squeezing" mechanism - as shown by the added thick lines (slope -2.4), the spectra are in fact very simple to explain with the anisotropic turbulence mechanism described here, indeed the transition point (10 and $100 \mathrm{~km}$ for meridional and zonal components 


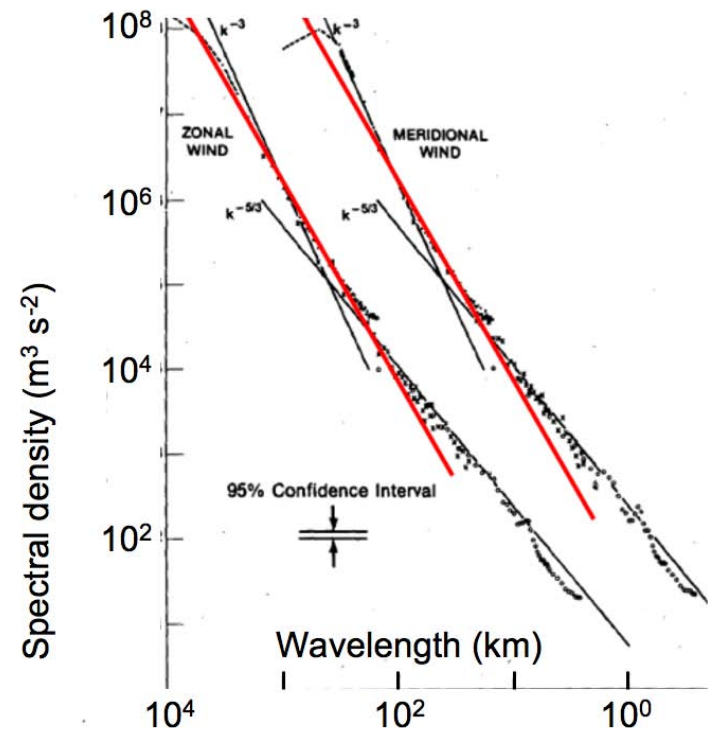

Fig. 7a. Key GASP results adapted from Gage and Nastrom (1986) with data broken into three groups depending on trajectory length (different symbols). It can be seen that the thick added reference lines with vertical exponent 2.4 fit very well from roughly 10 and $100 \mathrm{~km}$ on up (meridional and zonal components).

respectively) is quite close to the mean transition point found here (Fig. 3d, $40 \mathrm{~km}$ ). However, the spectrum shown in Fig. 7a is actually a composite of spectra with legs coming from three different length categories. When focusing on the larger scales it is thus more pertinent to focus on the longest leg category only; those longer than $4800 \mathrm{~km}$ (there is also less chance of statistical sample bias). When this is done (Fig. 7b), our reinterpretation is made all the more convincing since the large scale is seen to be nearly exactly of the predicted $k^{-2.4}$ form, with no plausible $k^{-3}$ regime whatsoever.

The GASP experiment involved commercial airliners flying along isobars near the top of the troposphere. It is therefore of interest to compare this with the Gao and Meriwether (1998) analysis of 11 legs of the scientific Electra aircraft which also flew along isobars (see Fig. $7 \mathrm{c}$ ) but at $\approx 6 \mathrm{~km}$. Concentrating on their spectra of horizontal wind, we find once again that exponents of 2.4 and $5 / 3$ with a transition at about $10 \mathrm{~km}$ explain the data very easily; the authors' overall regression estimate 1.98 (over the range 1-100 km) being a rough average of the two. Also, their regression giving a 3.18 exponent is only over the range $100-330 \mathrm{~km}$ and is not compelling. While our interpretation is fairly straightforward, the authors offer no explanation for their value 1.98 .

In the introduction, we mentioned that the most recent major campaign ( $>7600$ flights) was the MOZAIC campaign which - like the GASP experiment - also involved commercial aircraft flying along isobars (between 9.4 and $11.8 \mathrm{~km}$ ). Figure $7 \mathrm{~d}$ shows the second order structure function from Cho and Lindborg (2001). The spectral exponent $\beta=1+\xi(2)$ where $\xi(2)$ is the second order structure function exponent so

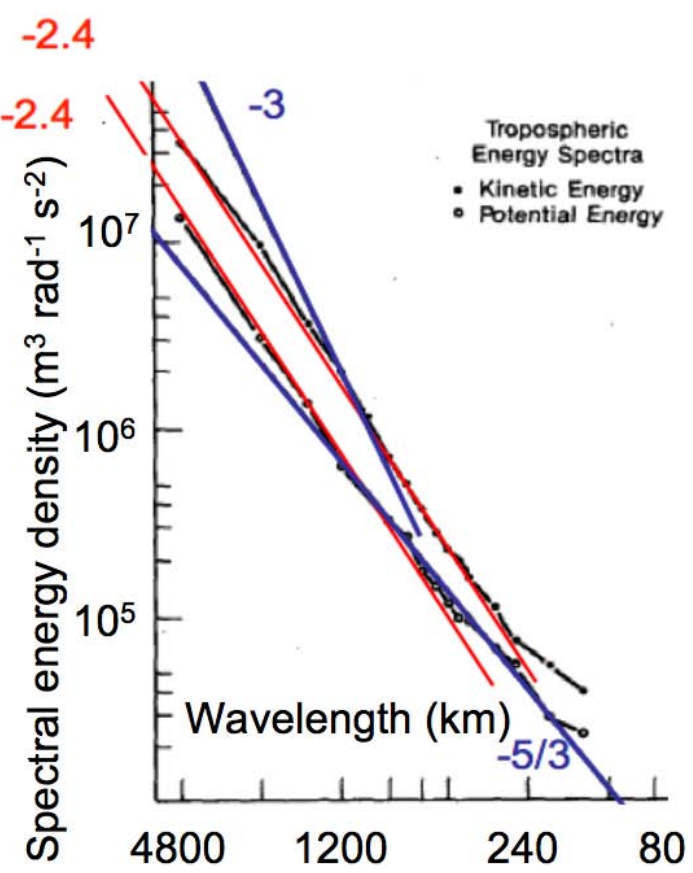

Fig. 7b. Adapted from Gage and Nastrom (1986) with the reference lines corresponding to the horizontal and vertical behaviour discussed in the text (slopes $-5 / 3,-2.4$, i.e. ignoring intermittency corrections corresponding to $H_{h}=1 / 3, H_{v}=0.7$ as well as the 2-D isotropic turbulence slope -3 ). This figure shows the spectra only for the particularly long legs (at least $4800 \mathrm{~km}$ long).

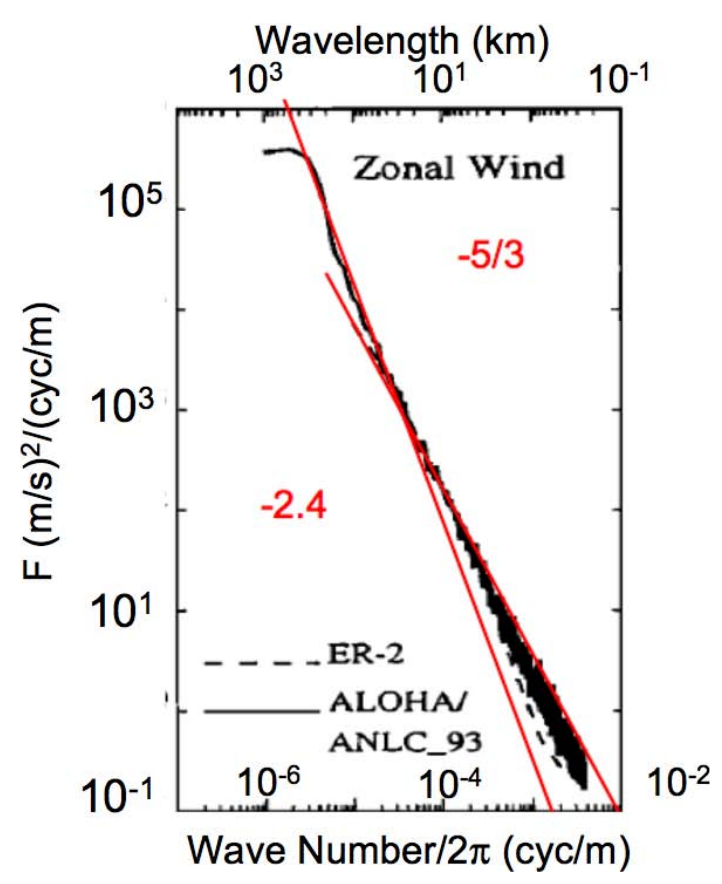

Fig. 7c. The averaged spectra adapted from Gao and Meriwether (1998) at $6 \mathrm{~km}$ altitude with the horizontal and vertical exponents discussed here indicated as reference lines. 


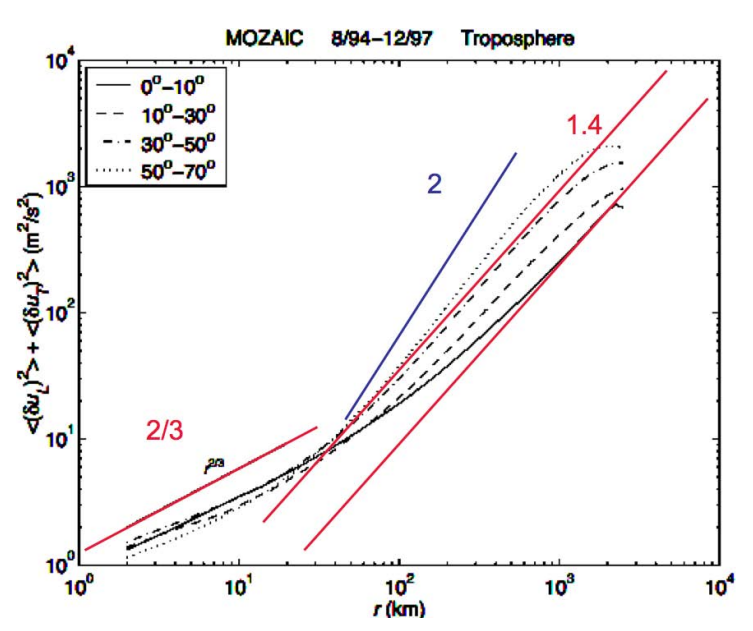

Fig. 7d. Adapted from Lindborg and Cho (2001): second order structure functions of the horizontal wind (sum of longitudinal and transverse components). Since the spectrum is (essentially) the fourier transform of the structure functions, the spectral behaviour $k^{-\beta}$ corresponds to $r^{(\beta-1)}$, hence the corresponding reference lines.

that the vertical exponent $\beta=2.4$ corresponds to $\xi(2)=1.4$. In the figure we see that our picture of a transition from horizontal exponent $2 / 3$ to vertical exponent 1.4 accurately accounts for the data over all the range (except for the extreme factor of 2-3 where the structure function levels off, a typical symptom of poor statistics) with the transition occurring at about $50 \mathrm{~km}$ i.e. about the same scale as in Fig. 3c. Also shown is a reference line $r^{2}$ which is the basic prediction of 2-D turbulence. Given the divergence of their curve from the $r^{2}$ line, it is surprising that Cho and Lindborg (2001) nevertheless claimed support for a 2-D isotropic turbulence regime. They did this by adding in a log correction. While such a correction is theoretically predicted in pure 2-D (Kraichnan) theory, it is normally considered a small effect and ignored. However Cho and Lindborg's claim to be able to save the 2-D theory by using log corrections was seriously undermined in the Lilley et al. (2008) reanalysis. They showed that the price paid in using $\log$ corrected $r^{2}$ law to explain a (near) $r^{1.4}$ law over an order of magnitude in scale is that the corrections must be so large as to imply impossible negative variances for scales $\approx 4000 \mathrm{~km}$ and larger.

In the introduction, we mentioned the stratospheric analyses Lovejoy et al. (2004) obtained from ER-2 aircraft following isomachs rather than isobars which found fractal trajectories with somewhat higher fractal dimensions $(\approx 1.55)$ than those found here $(\approx 1.33)$. These results are quite similar to those of Bacmeister et al. (1996) (Fig. 7e). Again, we see that over the analyzed range $0.4-102 \mathrm{~km}$ the data follows the slopes 5/3 and 2.4 quite well. In Bacmeister et al. (1996) exponents were estimated scale by scale and leg by leg so that histograms can be built up. While at the small scales, the mean exponent is near $\approx 5 / 3$, at the larger scales, as predicted, this value increases to about 2.5 .

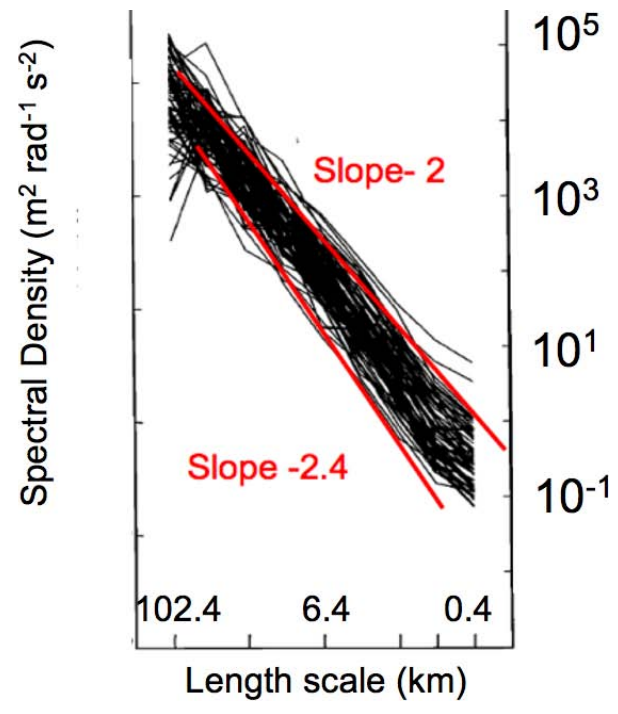

Fig. 7e. Stratospheric ER-2 spectra adapted from Bacmeister et al. (1996), Fig. 5. This is a random subset of $1024 \mathrm{~s}$ long legs, again with reference slopes added.

If our interpretation is correct, then there is a basic difference in the scaling exponents of the wind following isoheights and those following isobars (the latter being essentially equivalent to vertical exponents). This would help explain the wind spectra of atmospheric reanalyses where the value $\beta \approx 2.4$ has also been obtained (Stolle, 2009), along with a similar value in the vertical.

\section{Conclusions}

\subsection{Discussion}

In this paper, we examine in detail the characteristics of 16 horizontal tropospheric aircraft legs with an aim to systematically determining the consequences of the anisotropic turbulence on the vertically fluctuating trajectories. By calculating the mean absolute slopes as functions of scale, we discovered that for $\Delta x>3 \mathrm{~km}$ there is a significant intermediate fractal regime with $\Delta z \approx \Delta x^{H_{t r}}$, with $H_{t r} \approx 1 / 3$ followed (usually) at large $\Delta x$ by a transition to a regime with a mean constant slope, i.e. $H_{t r}=1$. At scales $\Delta x<3 \mathrm{~km}$ the slopes were lower than one would expect from an extrapolation from the fractal regime; presumably a consequence of the aircraft inertia. We argued that while the fractal regime was presumably dominated by turbulence, that the highly variable transition point from $H_{t r} \approx 1 / 3$ to $H_{t r} \approx 1$ depended on the level of turbulence, the slopes of the isobars and perhaps even the pilot and autopilot. By considering spectra and coherencies between the altitude and the wind and altitude $(z)$ and pressure $(p)$ measurements we showed that for most of the range of scales $>3 \mathrm{~km}$, that statistically significant coherencies and phase relations exist between $z$ and the longitu- 
dinal wind and even stronger coherencies and phase relations between $p$ and the longitudinal wind (and to much lesser degrees with the transverse wind). The longitudinal wind did however show significant coherencies in a narrower range $(\approx 10-30 \mathrm{~km})$. Since the aircraft did not fly in directions with special orientations the mere fact that the behaviour of the transverse and longitudinal wind was different in this regard supports the hypothesis that it is an artifact of the altitude/wind correlations. By examining the corresponding cross-spectral phases, we were able to show that these were also statistically significant over most of the range, with the wind fluctuations leading those of the altitude at the small $(<3 \mathrm{~km})$ aircraft inertial scales after which the altitude fluctuations lead the wind fluctuations up to $\approx 40 \mathrm{~km}$ followed by a further reversal corresponding precisely to the $k^{-2.4}$ range of the average spectrum, this time presumably due to meteorological correlations between the slopes of the isobars and the wind. The relation between the pressure and the wind was even stronger and clearer: at scales less than $\approx 40 \mathrm{~km}$ the pressure fluctuations lagged behind the wind fluctuations (especially the longitudinal wind) with a complete reversal at larger scales with the wind lagging behind the pressure. The intimate relation between altitude, pressure and wind statistics over even larger distances makes our reinterpretation compelling. In future, robotic aircraft ought to be fitted out so that they record the inputs and outputs to the autopilot. That way the aircraft motion relative to the atmosphere could, at least in principle, be solved as a problem in Newtonian physics and the relation of aircraft altitude to the meteorology could further clarified.

Using a "mean field" approximation (which ignores the correlation between the vertical fluctuations and the wind), we showed that for the trajectory fluctuations $\Delta z(\Delta x) \approx$ $\Delta x^{H_{t r}}$, there is a critical exponent $H_{z}$ such that if $H_{t r}<H_{z}$ then the turbulence will not affect the scaling of the horizontal wind, i.e. we continue to find $\Delta v \approx \Delta x^{H_{h}}$, while for $H_{t r}>H_{z}$ there will be a spurious break with the vertical exponent dominating for large $\Delta x$ where $\Delta v \approx \Delta x^{H_{v}}$. The prediction that there will be two scaling regimes separated by a break was directly tested using the ensemble statistics; it was found to hold with high accuracy with $H_{h} \approx 0.27 \pm 0.10$, $H_{v} \approx 0.66 \pm 0.07$. Due to the large uncertainties, we took this as confirmation that the Kolmogorov value $H_{h} \approx 1 / 3$ was correct, and we noted that the vertical value is somewhat biased towards small values since most but not all trajectories had a $\Delta x^{H_{v}}$ dominated regime. Fixing $H_{h}=1 / 3$ lead to the refined estimate $H_{z}=H_{h} / H_{v}=0.455 \pm 0.05$ implying $H_{v} \approx 0.73$ which is very close to the drop sonde estimate 0.75 for the $12-13 \mathrm{~km}$ level.

While these ensemble analyses supported the basic picture, new insight was obtained by looking at the legs individually. In particular, it was found that the transition point $\Delta x_{c}$ separating the $\Delta x^{H_{h}}$ and $\Delta x^{H_{v}}$ regimes was itself highly variable - in two cases being larger than the length of the trajectory (at least several hundreds of kilometers) while in some instances it was smaller, of the order of 10 kilometers. This high variability was itself predicted on the basis of the observed slope statistics, the high intermittency of the turbulence and the consequence of following isobars rather than constant altitudes. It goes a long way to explaining the plethora of horizontal scale breaks reported in the literature. It seems that at scales smaller than $\approx 40 \mathrm{~km}$ (but larger than an inertial scale of $\approx 3 \mathrm{~km}$ ) the aircraft follows turbulence driven fractal trajectories yet is nonetheless able to estimate the isoheight (horizontal) exponents whereas at larger scales, the aircraft accurately follows isobars yielding the different isobaric exponent which itself is at least approximately equal to the vertical exponent.

As a final test, we considered the predictions of the theory for the scale invariant lags $\zeta=\Delta x / \Delta z^{1 / H_{z}}$; this method takes into account the detailed vector fluctuation $\Delta r=(\Delta x$, $\Delta z$ ), i.e. the joint horizontal and vertical displacements of the aircraft. In this case the theoretical predictions were verified over 8 orders of magnitude in $\zeta$. It enabled us to rule out the possibility that there is a genuine large scale isotropic turbulent regime with exponent $H_{v}=H_{h} \approx 0.7-0.75$, since the longest flattest displacements followed $H_{h}=1 / 3$ very accurately. In addition the method allowed us to estimate the energy flux $\varepsilon$ and the sphero-scale $l_{s}$. Both values were found to be quite plausible given the published determinations in other experiments, notably the sphero-scale - the scale at which typical structures are roundish (structures become increasingly flat at larger scales) - was found to be in the range $20 \mathrm{~cm}$ to $2 \mathrm{~m}$, very close to the ER-2 estimate $(4 \mathrm{~cm})$ and the lidar estimate $(10 \mathrm{~cm}-80 \mathrm{~cm})$. As a general matter this scale invariant lag technique could profitably be used to remove the effect of the vertical fluctuations for analyzing other atmospheric fields. This will be developed further elsewhere.

\subsection{Implications for our understanding of the atmosphere}

The last thirty years has seen such a vast improvement in our ability to measure, analyze and model the atmosphere that at first sight it is incredible that there is still no consensus about its most basic statistical characteristics including the way that wind fluctuations vary with scale. Closer consideration however shows that no matter how precise or plentiful our measurements may be, that they nevertheless require theories, models and assumptions for their interpretation. The use of aircraft data in understanding the structure of the atmosphere provides a sobering illustration of this dialectic.

It now seems that the interpretations have typically been naïve: they have either simply ignored the vertical motion of the aircraft or have assumed that the turbulence is isotropic so that the vertical fluctuations do not strongly affect the statistics. When the analyses show breaks in the horizontal scaling (as they invariably do), rather than question the isotropy assumption and reinterpret the data, scientists tend to casually invoke the existence of two or more horizontal scaling 
regimes. The break between them is typically attributed to a transition from small scale three dimensional isotropic turbulence to large scale two dimensional isotropic turbulence and this, even if the break point varies considerably from experiment to experiment, and even if it is much bigger than the atmospheric scale height of $10 \mathrm{~km}$. However - as pointed out by Schertzer and Lovejoy (1985b) the very existence of such a "dimensional transition" (once called a "meso-scale gap", Van der Hoven, 1957) is itself only a theoretical consequence of the a priori assumption that turbulence must be isotropic! If the turbulence is anisotropic but scaling, then structures simply become flatter and flatter at larger and larger scales in a power law manner and such a transition is unnecessary. This is indeed the strong conclusion of a recent massive planetary scale study of short and long wave radiances (Lovejoy et al., 2009a). In this way, we see that the entire mainstream view of the atmosphere has fundamentally been coloured by the assumption of isotropic turbulence.

Cracks in this isotropic edifice started to appear in the 1980's when evidence started to mount that the key horizontal wind field has vertical statistics - including the scaling exponents - that were very different from those in the horizontal, suggesting that isotropic turbulence might be irrelevant to atmospheric dynamics. Incredibly, a recent literature review (Lilley et al., 2008) failed to find a single experimental study of the vertical which claimed evidence for the Kolmogorov scaling exponent $H_{v}=1 / 3$ - at any location or at any scale. On the contrary for twenty years, the debate among experimentalists on the vertical statistics has been between the values 3/5 (Bolgiano-Obukhov), 1 (quasi-linear gravity waves), and now with the more precise drop - sonde estimates, $H_{v}=0.60-0.75$ (low to high altitudes, still not well understood, Lovejoy et al., 2007). If this interpretation is correct, then it brings into question a number of phenomenologically based ideas including our ideas of convection. The appendix briefly discusses how the latter can be reconciled with wide range horizontal scaling. For more on this debate, see the online discussion.

The implications of these anisotropic scalings have not yet been translated into a proper understanding of the influence of vertical aircraft fluctuations nor into the interpretation of their measurements, nor into their significance for our overall understanding of the atmosphere. However, given the persistently central role played by isotropic theories of turbulence, the ramifications may take many years to fully discern.

\section{Appendix A}

\section{A1 Reconciling wide range scaling and convection}

Atmospheric convection is classically modelled by "twoscale" meteorological theories which typically single out a "convective scale" somewhere near the tropospheric scale height $(\approx 10-100 \mathrm{~km}$, e.g. Malkus and Reihl, 1958; Yano,
2009 , comment in the discussion version of this paper). In our analysis of the aircraft data we did find evidence for a scale break in the horizontal wind in this range - but we argued that it was a spurious consequence of the aircraft flying on isobars rather than on isoheights. In this appendix, we outline how this reinterpretation is compatible with convective phenomenology and convective cloud data from CloudSat, we also summarize the more detailed online response to Yano's comments (indicated Reply) below.

The reinterpretation is based on a model in which the underlying dynamical processes are anisotropic multiplicative cascades with different exponents in the horizontal and vertical directions. This has two consequences. First, that structures will systematically change shape/morphology with scale; in this case going from vertically oriented "cells" at small scales to flattened strata at large scales. Second, the fields will have strong singularities (hence coherent structures) distributed over sparse fractal sets (multifractals). With the help of simulations, we first illustrate how this explanation is compatible - at least in principle - with convective phenomenology, then, using CloudSat reflectivities (convection surrogates) where show that the latter do indeed respect anisotropic cascades.

\section{A2 The phenomenological fallacy}

Figure A1 gives an illustrative example of anisotropic cascade processes, with (roughly) the observed cascade parameters, yet each with a sphero-scale $l_{s}$ decreasing by factors of 4 corresponding to zooming out at random locations. One can see from the vertical cross-section (bottom row) that the degree of vertical stratification increases from left to right. These passive scalar cloud simulations (liquid water density bottom two rows, single scattering radiative transfer, top row) show that by zooming out (left to right) diverse morphologies appear. Although a phenomenologist might be tempted to introduce more than one mechanism to explain the morphologies at different scales in the figure we simply seeing the consequence of single underlying mechanism repeating scale after scale. In Lovejoy and Schertzer (2007) we call such inferences of mechanism from phenomena the "phenomenological fallacy". We argue that two-scale theories of convection are incompatible with the data which is scaling, and that the division into qualitatively distinct small and large regimes is unwarranted.

\section{A3 CloudSat estimates of horizontal-vertical relations}

Rather than speculate about the possible relation between horizontal and vertical scales in convective systems, we can use CloudSat reflectivities ( $Z$; a convection surrogate) to determine the relationship directly. Indeed, the fluctuations $\Delta Z$ implicitly determine horizontal/vertical relations. For example, defining $\Delta Z$ as the absolute difference over a horizontal lag $\Delta x$ or a vertical lag $\Delta z$, (or absolute wavelet coefficient) 

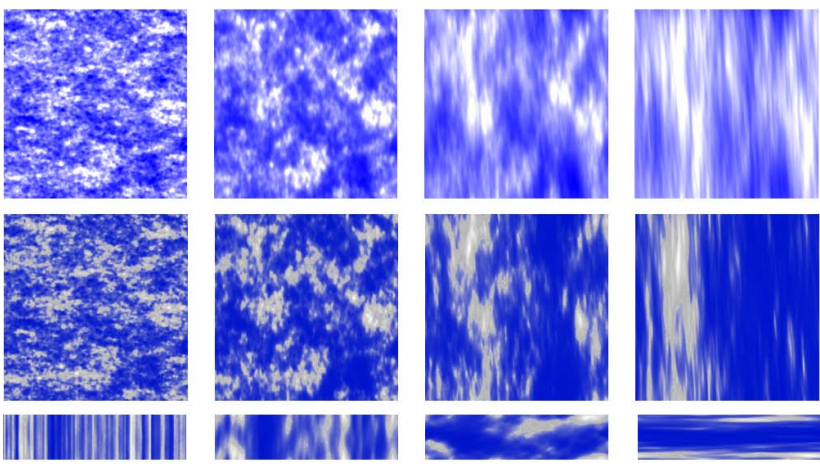

Fig. A1. Examples of continuous in scale anisotropic multifractals in 3 -D $(256 \times 256 \times 64)$. The effect of changing the sphero-scale $\left(l_{s}\right.$, see Sect. 3) on multifractal models of clouds with $H_{z}=5 / 9$. The cloud parameters are: $\alpha=1.8, C_{1}=0.1, H=1 / 3$ (similar to CloudSat, aerosols, see below). From left to right decreasing $l_{s}$ corresponding to zooming out by factors of 4 so that the we see the initially vertically aligned structures (bottom left) becoming quite flat at scales 64 times larger (right). At the same time, the horizontal structures have anisotropy characterized by the matrix $\mathbf{G}=((0.8$, $-0.02),(0.02,1.2))$ so that they too change orientation, elongation (the horizontal sphero-scale starts at 1 pixel, far left). The middle row is false colour of the liquid water density field, the bottom row is the corresponding vertical sections (side view), the top row is the corresponding single scatter visible radiation, the mean optical thickness is 2 , isotropic scattering phase function, sun incident at $45^{\circ}$ to the right.

for each horizontal extent $\Delta x$ we can find the corresponding vertical extent $\Delta \mathrm{z}(\Delta \mathrm{x})$ by solving the implicit equation for the ensemble averaged fluctuations:

$$
\langle\Delta Z(\Delta z)\rangle=\langle\Delta Z(\Delta x)\rangle .
$$

Figure A2 shows the result on 16 CloudSat orbits, for fluctuations defined from orbit by orbit averages as well from an ensemble average over all the orbits. The mean of the individual orbit by orbit $\Delta z(\Delta x)$ curves and the ensemble are nearly identical; the orbit to orbit spread is shown as one standard deviation curves above and below (the curves are occasionally double-valued along the $\Delta x$ axis due to statistical fluctuations). In addition to the empirical curves, we have provided two theoretical reference lines with slopes $H_{z}=5 / 9$, 1. The former is the prediction of the 23/9 D model of atmospheric dynamics discussed in section 3 and accurately confirmed for aerosols in Lilley et al. (2004), the intersection of two lines is the "sphero-scale" i.e. the scale where the structures are roundish, here on average at about $100 \mathrm{~m}$ (incidentally, the bisectrix is the prediction of isotropic 3-D turbulence). Structures at larger scales are flat, while at smaller scales they are elongated in the vertical. Although the exponents for $Z$ and for lidar aerosol backscatter are nearly identical (given by the theoretical anisotropic Corrsin-Obhukov values), the corresponding sphero-scales $l_{s}$ are about a factor 1000 larger (although as can be seen from the error,

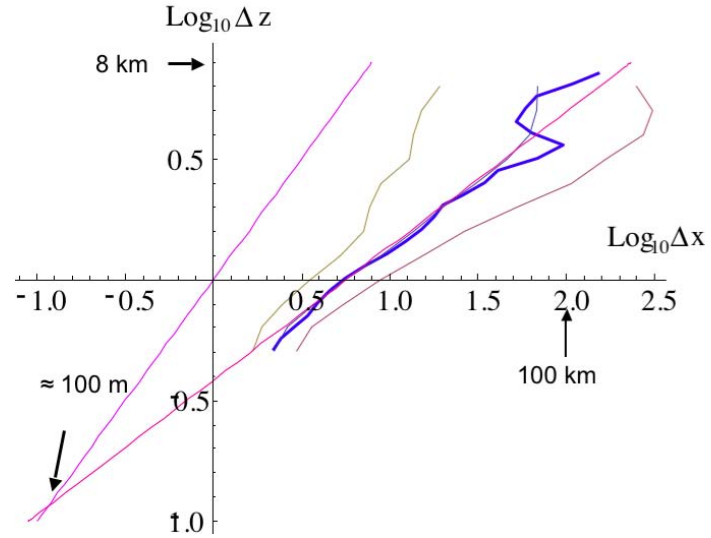

Fig. A2. A space (horizontal) - space (vertical) diagramme estimated from the absolute reflectivity fluctuations from 16 CloudSat orbits. Reference lines have slopes $1,5 / 9$, see text.

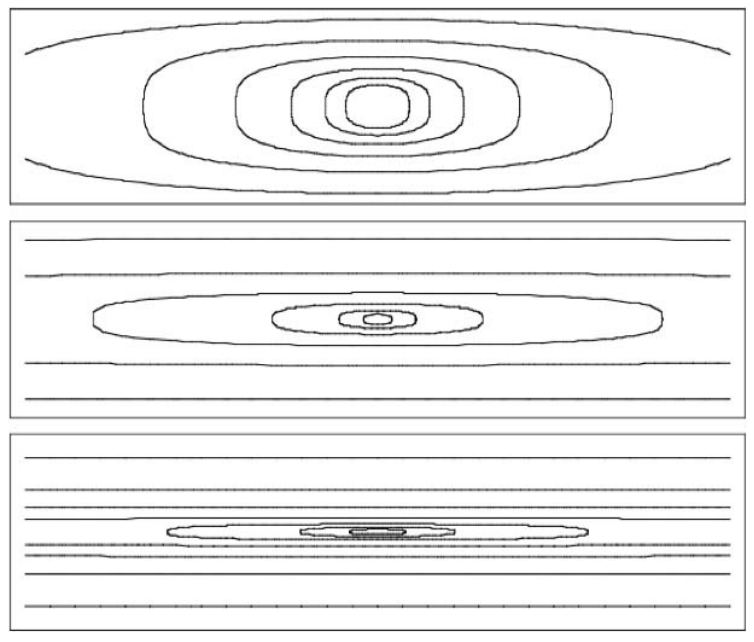

Fig. A3. This shows the theoretical shapes of average vertical cross-sections using the CloudSat derived mean parameters from Fig. 2: $H_{z}=5 / 9$, with sphero-scales $1 \mathrm{~km}$ (top), $100 \mathrm{~m}$ (middle), $10 \mathrm{~m}$ (bottom), roughly corresponding to the geometric mean and one standard deviation fluctuations. The distance from left to right is $100 \mathrm{~km}$, from top to bottom is $20 \mathrm{~km}$. It uses the canonical scale function given in Eq. (7). The top figure in particular shows that structures $100 \mathrm{~km}$ wide will be about $10 \mathrm{~km}$ thick whenever the sphero-scale is somewhat larger than average.

there is a large scatter; the mean of $\log _{10} l_{s}$ with $l_{s}$ in $\mathrm{km}$ is $\approx-1.6 \pm 0.9$ i.e. one standard deviation bars are $5 \mathrm{~m}$ to $500 \mathrm{~m}$, geometric mean $50 \mathrm{~m}$ ). Figure A3 shows the corresponding average contours of cloud reflectivity structures showing how they very gradually tend to rounder shapes at the larger scales.

Acknowledgements. We acknowledge NOAA funding of the Winter Storms 2004 mission. This research was performed purely for scientific purposes, it did not enjoy any specific funding.

Edited by: P. Spichtinger 


\section{References}

Adelfang, S. I.: On the relation between wind shears over various intervals, J. Appl. Meteorol., 10(10), 156-159, 1971.

Bacmeister, J. T., Eckermann, S. D., Newman, P. A., Lait, L., Chan, K. R., Loewenstein, M., Proffitt, M. H., and Gary, B. L.: Stratospheric horizontal wavenumber spectra of winds, potnetial temperature, and atmospheric tracers observed by high-altitude aircraft, J. Geophys. Res., 101, 9441-9470, 1996.

Cho, J. and Lindborg, E.: Horizontal velocity structure functions in the upper troposphere and lower stratosphere 1. Observations, J. Geophys. Res., 106(D10), 10223-10 232, 2001.

Dewan, E. and Good, R.: Saturation and the "universal" spectrum vertical profiles of horizontal scalar winds in the stratosphere, J. Geophys. Res., 91, p. 2742, 1986.

Dewan, E.: Saturated-cascade similtude theory of gravity wave spectra, J. Geophys. Res. , 102, 29 799-29 817, 1997.

Endlich, R. M., Singleton, R. C., and Kaufman, J. W.: Spectral Analyes of detailed vertical wind profiles, J. Atmos. Sci., 26, 1030-1041, 1969.

Fritts, D. and Chou, H.: An investigation of the vertical wavenumber and frequency spectra of gravity wave motions in the lower stratosphere, J. Atmos. Sci., 44, p. 3611, 1987.

Gage, K. S. and Nastrom, G. D.: Theoretical Interpretation of atmospheric wavenumber spectra of wind and temperature observed by commercial aircraft during GASP, J. Atmos. Sci., 43, 729$740,1986$.

Gao, X. and Meriwether, J. W.: Mesoscale spectral analysis of in situ horizontal and vertical wind measurements at $6 \mathrm{~km}$, J. Geophys. Res., 103, 6397-6404, 1998.

Gardner, C.: Diffusive filtering theory of gravity wave spectra in the atmosphere, J. Geophys. Res., 99(D10), 20 601, 1994.

Gardner, C., Tao, X., and Papen, G.: Simultaneous lidar observations of vertical wind, tmepreature and density profiles in the upper atmsophere: evidence of nonseperability of atmospheric perturbation spectra, Geophys. Res. Lett., 22(20), p. 2877, 1995.

Gardner, C. S., Hostetler, C. A., and Franke, S. J.: Gravity Wave models for the horizontal wave number spectra of atmospheric velocity and density flucutations, J. Geophys. Res., 98, 10351049, 1993.

Hovde, S. J., Tuck, A. F., Lovejoy, S., and Schertzer, D.: Vertical Scaling of the Atmosphere: Dropsondes from $13 \mathrm{~km}$ to the Surface, Q. J. Royal Meteor. Soc., in review, 2009.

Koch, S. E., Jamison, B. D., Lu, C. G., Smith, T. L., Tollerud, E. I., Girz, C., Wang, N., Lane, T. P., Shapiro, M. A., Parrish, D. D., and Cooper, O. R.: Turbulence and gravity waves within an upper-level front, J. Atmos. Sci., 62, 3885-3908, 2005.

Landahl, M. T. and Mollo-Christensen, E.: Turbulence and random processes in fluid mechanics, Cambridge University press, Cambridge, 154 pp., 1986.

Lazarev, A., Schertzer, D., Lovejoy, S., and Chigirinskaya, Y.: Unified multifractal atmospheric dynamics tested in the tropics: part II, vertical scaling and generalized scale invariance, Nonlin. Processes Geophys., 1, 115-123, 1994, http://www.nonlin-processes-geophys.net/1/115/1994/.

Lilly, D. K.: Two-dimensional turbulence generated by energy sources at two scales, J. Atmos. Sci., 46, 2026-2030, 1989.

Lilley, M., Lovejoy, S., Strawbridge, K., and Schertzer, D.: 23/9 dimensional anisotropic scaling of passive admixtures using lidar aerosol data, Phys. Rev. E, 70(3), 7 pp., 2004.

Lilley, M., Lovejoy, S., Schertzer, D., Strawbridge, K. B., and Radkevitch, A.: Scaling turbulent atmospheric stratification, Part II: empirical study of the the stratification of the intermittency, Q. J. Roy. Meteor. Soc., 134(631), 301-315, 2008.

Lindborg, E.: Can the atmospheric kinetic energy spectrum be explained by two-dimensional turbulence?, J. Fluid Mech., 388, 259-288, 1999.

Lindborg, E. and Cho, J.: Horizontal velocity structure functions in the upper troposphere and lower stratosphere 2. Theoretical considerations, J. Geophys. Res., 106, 10 233-10 241, 2001.

Lovejoy, S., Schertzer, D., and Tuck, A. F.: Fractal aircraft trajectories and nonclassical turbulent exponents, Phys. Rev. E, 70(3), 5 pp., 2004.

Lovejoy, S. and Schertzer, D.: Scale, scaling and multifractals in geophysics: twenty years on, in: Nonlinear dynamics in geophysics, edited by: Tsonis, A. A. and Elsner, J., Elsevier, 2007.

Lovejoy, S., Tuck, A. F., Hovde, S. J., and Schertzer, D.: Is isotropic turbulence relevant in the atmosphere?, Geophys. Res. Lett., 34, L14802, doi:10.1029/2007GL029359., 2007.

Lovejoy, S., Schertzer, D., Lilley, M., Strawbridge, K. B., and Radkevitch, A.: Scaling turbulent atmospheric stratification, Part I: Turbulence and waves, Q. J. Roy. Meteor. Soc., 134(631), 277300, 2008.

Lovejoy, S., Schertzer, D., Allaire, V., Bourgeois, T., King, S., Pinel, J., and Stolle, J.: Atmospheric complexity or scale by scale simplicity? , Geophys. Res. Lett., 36, L01801, doi:01810.01029/02008GL035863, 2009a.

Lovejoy, S., Tuck, A., and Schertzer, D.: The Horizontal cascade structure of atmospheric fields determined from aircraft data, J. Geophys. Res., in review, 2009b.

Lovejoy, S., Tuck, A. F., Hovde, S. J., and Schertzer, D.: The vertical cascade structure of the atmosphere and multifractal drop sonde outages, J. Geophys. Res., 114, D07111, doi:07110.01029/02008JD010651., 2009c.

Nastrom, G. D. and Gage, K. S.: A first look at wave number spectra from GASP data, Tellus A, 35, p. 383, 1983.

Nastrom, G. D., Gage, K. S., and Jasperson, W. H.: Kinetic energy spectrum of large and meso-scale atmospheric processes, Nature, 310, 36-38, 1984.

Nastrom, G. D. and Gage, K. S.: A climatology of atmospheric wavenumber spectra of wind and temperature by commercial aircraft, J. Atmos. Sci., 42, 950-960, 1985.

Radkevitch, A., Lovejoy, S., Strawbridge, K. B., Schertzer, D., and Lilley, M.: Scaling turbulent atmospheric stratification, Part III: Space-time stratification of passive scalars using lidar data, Q. J. Roy. Meteor. Soc., 134(631), 317-335, 2008.

Ray, E. A., Rosenlof, K. H., Richard, E., Parrish, D., and Jakoubek, R.: Distributions of ozone in the region of the subtropical jet: An analysis of in situ aircraft measurements, J. Geophys. Res.Atmos., 109, D08106, doi:10.1029/2003JD004143, 2004.

Schertzer, D. and Lovejoy, S.: Generalised scale invariance in turbulent phenomena, Physico-Chemical Hydrodynamics Journal, 6, 623-635, 1985a.

Schertzer, D. and Lovejoy, S.: The dimension and intermittency of atmospheric dynamics, in: Turbulent Shear Flow 4, edited by: Launder, B., Springer-Verlag, 7-33, 1985b.

Stolle, : Space-time Cascade structure of numerical models of the atmosphere, McGill, Montreal, 2009. 
Tsuda, T., Inoue, T., Fritts, D., VanZandt, T., Kato, S., Sato, T., and Fukao, S.: MST radar observations of a saturated gravity wave spectrum, J. Atmos. Sci., 46, 2440, 1989.

Tuck, A.: ATMOSPHERIC TURBULENCE: A Molecular Dynamics Perspective, Oxford University Press, 2008.
Van der Hoven, I.: Power spectrum of horizontal wind speed in the frequency range from .0007 to 900 cycles per hour, J. Meteorol., 14, 160-164, 1957.

Van Zandt, T. E.: A universal spectrum of buoyancy waves in the atmosphere, Geophys. Res. Lett., 9, 575-578, 1982. 